\title{
Decision-Directed Multivariate Empirical Bayes Classification with Nonstationary Priors
}

\author{
WYNN C. STIRLING AND A. LEE SWINDLEHURST
}

\begin{abstract}
A decision-directed learning strategy is presented to recursively estimate (i.e., track) the time-varying a priori distribution for a multivariate empirical Bayes adaptive classification rule. The problem is formulated by modeling the prior distribution as a finite-state vector Markov chain and using past decisions to estimate the time evolution of the state of this chain. The solution is obtained by implementing an exact recursive nonlinear estimator for the rate vector of a multivariate discrete-time point process representing the decisions. This estimator obtains the Doob decomposition of the decision process with respect to the $\sigma$-field generated by all past decisions and corresponds to the nonlinear least squares estimate of the prior distribution. Monte Carlo simulation results are provided to assess the performance of the estimator.
\end{abstract}

Index Terms-Adaptive classification, decision-directed learning, discrete-time point process, empirical Bayes, Markov chains, martingale difference processes, multivariate detection.

\section{INTRODUCTION}

$\mathrm{T}$ HIS paper addresses the problem of learning the prior probability of occurrence (i.e., relative frequency) of a signal or class of signals with respect to the parameters of the classification system. This statistical representation of the signal occurrences leads to the development of decision techniques which may be tuned to the local characteristics of the signal and permit on-line tracking or learning of these characteristics. We pursue an empirical Bayes approach, wherein the prior distribution is estimated from the data. Empirical Bayes procedures are well known, [1]-[4], but deal almost exclusively with the stationary case where the prior distribution is constant. For the stationary case, there exist asymptotically subminimax decision rules that approach the Bayes envelope. Our problem is somewhat more complex, however, since we permit the decision environment to be nonstationary and assume that the prior distribution may evolve with time. One may not be able to wait until all the data are received to make decisions. In fact, a real-time classification capability is often necessary, and critically, it must be able to adjust the structure of the decision rule adaptively to ensure that the classification is being made as accurately as possible. These constraints on the empirical Bayes procedure are severe and render classical decision rules inadequate to deal with the tracking capability. As an alternative, the decision-directed approach represents a

Manuscript received June 23, 1986; revised January 8, 1987. Recommended for acceptance by J. Kittler.

The authors are with the Department of Electrical Engineering, Brigham Young University, Provo, UT 84602.

IEEE Log Number 8715815. significant departure from classical empirical Bayes procedures, but fits well into the general class of adaptive detection procedures such as generalized likelihood ratio tests.

The philosophy of decision-directed procedures is illustrated in Fig. 1. In this figure, we consider a received signal that is then subjected to processing, which involves decision-making procedures to detect and extract the signal. The outputs of the signal processor may be used to generate an estimate of the signal model and feed it back into the classification block to modify the decision rule.

The problem of simultaneous detection and estimation is developed in a decision-theoretic setting in [5], [6], although these researchers do not discuss the decision-theoretic extensions to the problem. Decision-directed procedures have been used in [7]-[10] for adaptive (untaught) pattern recognition, wherein signal parameters are estimated using only observations classified as containing the signal. Other decision-directed adaptive classification schemes have been proposed for synchronous detection [11] and for adaptive equalization in the digital communications context [12]. Decision-directed procedures have been used for various unsupervised learning problems [13]-[20] and for analysis of Gaussian mixtures [21]. Perhaps the first rigorous analysis of the decision-directed empirical Bayes approach was conducted in [22], which investigates the binary detection problem with unknown priors. These results have been further studied in [23][25]. Extension to the nonstationary case is provided in [26]-[28].

The espousal of the Bayesian approach implies that the unknown state of nature is described by a prior probability distribution. The empirical Bayes decision problem is formulated in exactly the same way as a standard Bayes problem, except that the prior is unknown and must be estimated from the available data. Suppose that a particular decision problem occurs repeatedly and independently, with the same unknown prior distribution throughout the experiment. Under this supposition, it is logical to perform analysis on the observation in an attempt to discover the prior distribution. We may define an empirical decision procedure as a sequence of decision rules which learn or adapt from previous experiments and "converge" in some sense to the true prior. Robbins and related researchers [1] describe the theory of asymptotically optimal decision procedures and demonstrate that such procedures converge to the Bayes envelope function as the number of experiments increases. These results are 


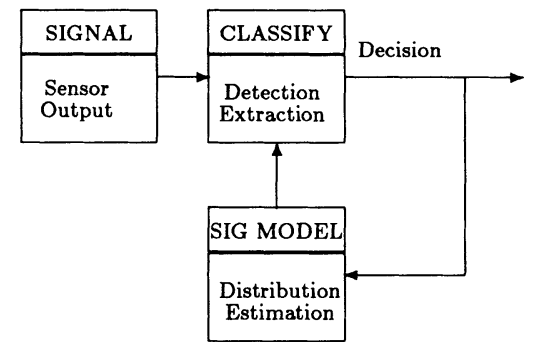

Fig. 1. Feedback, or decision-directed, rules.

based upon the assumption that the prior distribution is constant throughout the experiment.

A key aspect of this study is that the prior distribution is not only unknown, but is subject to change as well. This change in the assumptions about the prior constitutes a significant difficulty since the classical convergence results may no longer be valid. In the extreme case where the changes are completely unpredictable, it is likely that the empirical Bayes approach is doomed to failure, and some other decision criteria should be evoked. If the changes can be modeled, however, then there is hope that the prior distribution may be "tracked"' in a manner entirely analogous to the way a moving target is tracked using, say, a Kalman filter. The key assumption, therefore, is the model that is used to describe the evolution of the distribution. In this study, we develop such a model for the multivariate probability distribution function of a multiple hypothesis detection problem. This estimator is cast in a state-space environment (analogous to a differential system), and a recursive state estimator (analogous to a Kalman filter) for time-varying prior distribution is presented.

\section{Multivariate Distribution Estimation}

Let $\boldsymbol{Y} \in \mathbb{R}^{m}$ denote the signal space, and let $\boldsymbol{y}(t) \in \boldsymbol{Y}$ denote the observed signal at time $t$ for $t=0,1, \cdots$. Let $S$ denote a classification set for the signal $y$, and defined a decision function

$$
D: Y \mapsto\{0,1\}
$$

as a binary-valued function mapping the observed signal into an abstract classification space. That is,

$$
D[\boldsymbol{y}(t)]= \begin{cases}1 & S \text { occurs } \\ 0 & S \text { does not occur. }\end{cases}
$$

For $m$ classes $S_{i}, i=1, \cdots, m$, we may define

$$
D_{i}[y(t)]= \begin{cases}1 & S_{i} \text { occurs } \\ 0 & S_{i} \text { does not occur }\end{cases}
$$

and consider the $m$-vector decision function

$$
\boldsymbol{D}(\cdot)=\left[D_{1}(\cdot) \cdots D_{m}(\cdot)\right]^{T} \text {. }
$$

We note that the classification sets $S_{i}$ need not be disjoint (i.e., $\boldsymbol{y}(t)$ may belong to any or all of the classes). We will assume that $\boldsymbol{y}(t)$ must be classified into at least one of the $S_{i}$.

We are confronted with an $M$-ary decision problem ( $M$ $=2^{m}$ ) involving the $M$ hypotheses

$$
H_{\alpha_{1}} \cdots \alpha_{m}: S^{\alpha_{1} \cdots \alpha_{m}}, \quad \alpha_{1} \cdots \alpha_{m} \in\{0,1\}^{m}
$$

where

with

$$
\mathcal{S}^{\alpha_{1} \cdots \alpha_{m}}=S_{1}^{\alpha_{1}} \cap S_{2}^{\alpha_{2}} \cap \cdots \cap S_{m}^{\alpha_{m}}
$$

$$
S^{\alpha}= \begin{cases}S & \text { for } \alpha=1 \\ \bar{S} & \text { for } \alpha=0\end{cases}
$$

where $\bar{S}$ means that $S$ does not occur. The Bayesian approach to this problem is to choose the hypothesis $H_{i}$ for which the likelihood function

$$
\pi^{\alpha_{1} \cdots \alpha_{m}}(t) f\left(\boldsymbol{y}(t) \mid \mathcal{S}^{\alpha_{1} \cdots \alpha_{m}}\right)
$$

is maximized where $f\left(\boldsymbol{y}(t) \mid \mathcal{S}^{\alpha_{1} \cdots \alpha_{m}}\right)$ is the distribution of $\boldsymbol{y}(t)$ under classification $\mathcal{S}^{\alpha_{1} \cdots \alpha_{m}}$ and

$$
\pi^{\alpha_{1} \cdots \alpha_{m}}(t)=P\left\{\mathcal{S}^{\alpha_{1} \cdots \alpha_{m}}\right\}
$$

is the a priori probability distribution for each of the events $S^{\alpha_{1} \cdots \alpha_{m}}$. The decision-directed empirical Bayes approach is to estimate $\pi^{\alpha_{1}} \cdots \alpha_{m}(t)$ by means of the past decisions $\{\boldsymbol{D}(s), s<t\}$.

We wish to consider the problem of estimating the joint probability distribution of the vector decision process $\boldsymbol{D}(\cdot)$. In other words, if we define the discrete-time vector point process $N(t)=\left[N_{1}(t) \cdots N_{m}(t)\right]^{T}$ where

$$
\begin{aligned}
& N_{i}(t)=D_{i}\lceil y(t)\rceil, \\
& \quad i=1, \cdots, m, t=0,1, \cdots,
\end{aligned}
$$

then the problem is to estimate the probability mass function $p_{N(t)}(\boldsymbol{\alpha})=P\left\{N_{1}(t)=\alpha_{1}, \cdots, N_{m}(t)=\alpha_{m}\right\}$ for each $t$ where $\boldsymbol{\alpha}=\left[\alpha_{1} \cdots \alpha_{m}\right]^{T}$. In point of fact, $p_{N(t)}(\boldsymbol{\alpha})$ is the marginal distribution of the joint vector $N(0)$, $\boldsymbol{N}(1), \cdots$, but we will, for purposes of this analysis, be concerned only with these marginals and will investigate dynamical equations for them, rather than estimate the entire joint vector distribution. We will see that dynamical equations for $p_{N(t)}(\boldsymbol{\alpha})$ will be suitable for purpose of tracking real-time behavior of the process $N(t)$ whereas the joint distribution of the entire process will not be available in real time.

\section{A. Probability Models}

Let $\beta_{t-1}$ denote a $\sigma$-field generated by all of the factors that may affect the distribution of the process $N(t)$ at time $t$, and define the joint conditional probability mass function

$$
\begin{aligned}
& p_{N(t)}\left(\boldsymbol{\alpha} \mid \Re_{t-1}\right)= \\
& \quad P\left\{N_{1}(t)=\alpha_{1}, \cdots, N_{m}(t)=\alpha_{m} \mid \Re_{t-1}\right\} .
\end{aligned}
$$

This joint probability mass function may be represented in terms of $2^{m}=M$ conditional probabilities, or rates,

$$
\begin{aligned}
& \lambda^{\alpha_{1} \cdots \alpha_{m}}(t)=P\left\{\delta^{\alpha_{1} \cdots \alpha_{m}} \mid \bigotimes_{t-1}\right\} \\
& =P\left\{\prod_{i=1}^{m}\left[N_{i}^{\alpha_{i}}(t)\left(1-N_{i}(t)\right)^{1-\alpha_{i}}\right]=1 \mid \bigotimes_{t-1}\right\} \\
& \quad=E^{\Theta_{t-1}}\left\{\prod_{i=1}^{m} N_{i}^{\alpha_{i}}(t) \bar{N}_{i}^{\alpha_{i}}(t)\right\}
\end{aligned}
$$

where $\bar{N}$ and $\bar{\alpha}$ denote $1-N$ and $1-\alpha$, respectively, the superscript $\alpha$ on $N$ means that $N$ is raised to the power $\alpha$ (i.e., $N^{1}=N$ and $N^{0}=1$ ), and the notation $E^{\circledR}\{\cdot\}$ 
(or alternatively, $E\{\cdot \mid \beta)$ denotes the conditional expectation of the argument with respect to the $\sigma$-field $B$.

Let us define the vector $\Lambda(t)$ as

$$
\boldsymbol{\Lambda}(t)=\left[\begin{array}{c}
\lambda^{1 \cdots 111}(t) \\
\lambda^{1 \cdots 110}(t) \\
\lambda^{1 \cdots 101}(t) \\
\vdots \\
\lambda^{0 \cdots 011}(t) \\
\lambda^{0 \cdots 010}(t) \\
\lambda^{0 \cdots 001}(t)
\end{array}\right] 2^{m}-1 \text { vector terms. }
$$

Since the events are mutually exclusive, we have

$$
\sum_{\substack{\alpha_{1} \cdots \alpha_{m} \\ \text { some } \alpha_{i}=1}} \lambda^{\alpha_{1} \cdots \alpha_{m}}(t) \leq 1
$$

where the sum is taken over all $2^{m}-1$ values of $\alpha_{1} \cdots$ $\alpha_{m}$ such that at least one of the $\alpha_{i}$ is nonzero. Since the sum of all values of the probability mass function must equal unity (the simplex constraint), this constraint may

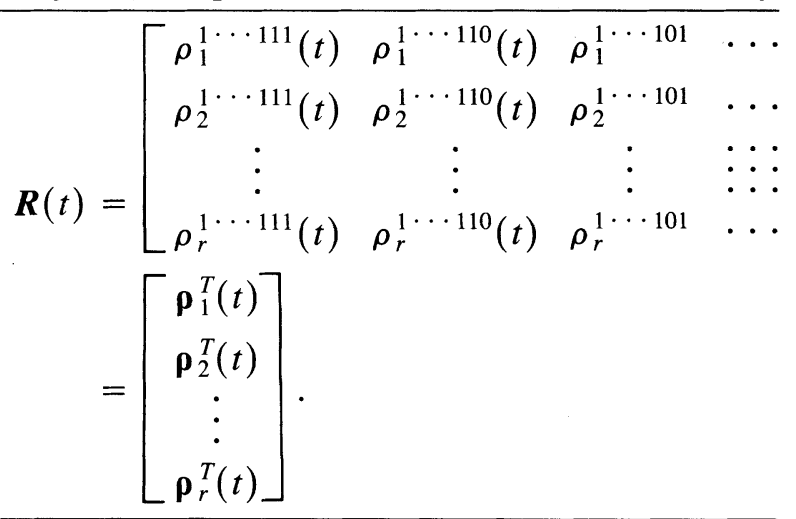

be guaranteed by defining

$$
\lambda^{0 \cdots 0}(t)=1-\sum_{\substack{\alpha_{1} \cdots \alpha_{m} \\ \text { some } \alpha_{i}=1}} \lambda^{\alpha_{1} \cdots \alpha_{m}}(t) .
$$

We wish to estimate $\boldsymbol{\Lambda}(t)$ as a function of time. To do this, we require a probabilistic model to characterize this vector and a model to describe its evolution in time. A physically meaningful, and at the same time mathematically tractable, model for $\boldsymbol{\Lambda}(t)$ is to represent it as a finite-state Markov chain. Such a model may be contrasted with a continuous model for $\boldsymbol{\Lambda}(t)$ as follows.

1) The Markov structure permits the evolution of $\boldsymbol{\Lambda}(t)$ to be treated probabilistically via the state transition matrix. This representation may be contrasted with a stochastic differential or difference equation for $\boldsymbol{\Lambda}(t)$, which may be difficult to treat analytically.

2) The finite-state model permits limits on the range of $\boldsymbol{\Lambda}(t)$ to be imposed, and the rate may be restricted to the expected domain of the parameter space. Such a limitation may, for example, be chosen to reduce or eliminate the probability of runaway (i.e., divergence of the estimator), which is a possibility in the decision-directed estimation context.
Under the Markov structure, we may represent $\boldsymbol{\Lambda}(t)$ as a finite-state vector Markov chain with states $\boldsymbol{\rho}_{1}(t)$, $\cdots, \boldsymbol{p}_{r}(t)$ where

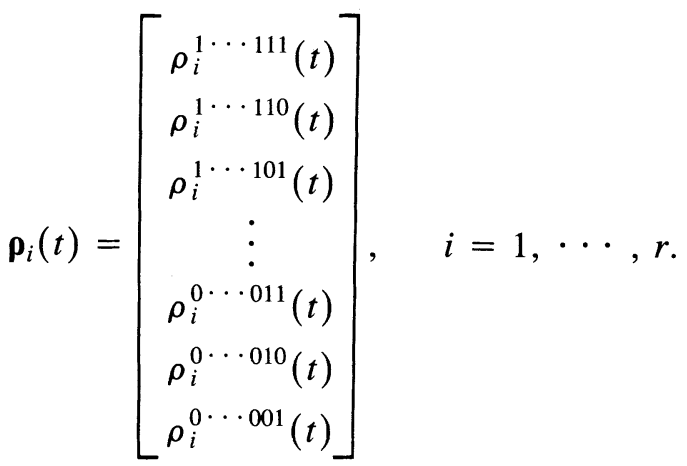

Define the state vector $\boldsymbol{x}(t)=\left[x_{1}(t), \cdots, x_{r}(t)\right]^{T}$ as

$$
x_{i}(t)=\left\{\begin{array}{ll}
1 & \text { if } \boldsymbol{\Lambda}(t)=\boldsymbol{\rho}_{i}(t) \\
0 & \text { otherwise, }
\end{array} \quad i=1, \cdots, r .\right.
$$

Thus,

$$
\mathbf{\Lambda}(t)=\boldsymbol{R}^{T}(t) \boldsymbol{x}(t)
$$

where the $r \times(M-1)$ matrix $R$ is

$$
\left.\begin{array}{cccc}
\rho_{1}^{0 \cdots 011}(t) & \rho_{1}^{0 \cdots 011}(t) & \rho_{1}^{0 \cdots 010}(t) & \rho_{1}^{0 \cdots 001}(t) \\
\rho_{2}^{0 \cdots 011}(t) & \rho_{2}^{0 \cdots 011}(t) & \rho_{2}^{0 \cdots 010}(t) & \rho_{2}^{0 \cdots 001}(t) \\
\vdots & \vdots & \vdots & \vdots \\
\rho_{r}^{0 \cdots 011}(t) & \rho_{r}^{0 \cdots 011}(t) & \rho_{r}^{0 \cdots 010}(t) & \rho_{r}^{0 \cdots 001}(t)
\end{array}\right]
$$

The state, therefore, characterizes the probability distribution of the vector process $N(t)$. In other words, knowledge of $\boldsymbol{x}(t)$ specifies the joint probability mass function $p_{N(t)}(\boldsymbol{\alpha})$ since we have

$$
p_{N(t)}\left(\alpha_{1} \cdots \alpha_{m}\right)=\lambda^{\alpha_{1} \cdots \alpha_{m}}(t)=\rho_{i}^{\alpha_{1} \cdots \alpha_{m}}(t)
$$

where the index $i$ corresponds to the single nonzero element of $\boldsymbol{x}(t)$.

The evolution of the state may be characterized by means of a state probability transition matrix

$$
\begin{gathered}
q_{i j}(t)=P\left\{x_{j}(t+1)=1 \mid x_{i}(t)=1\right\}, \\
i, j=1, \cdots, r .
\end{gathered}
$$

Let $\boldsymbol{Q}(t)=\left[q_{i j}(t)\right]$ denote the state transition matrix. Then the state evolves as

$$
\boldsymbol{x}(t+1)=\boldsymbol{Q}^{T}(t) \boldsymbol{x}(t)+\boldsymbol{u}(t)
$$

where $\boldsymbol{u}(t)=\boldsymbol{x}(t+1)-\boldsymbol{Q}^{T}(t) \boldsymbol{x}(t)$. Define the family of $\sigma$-fields

$$
B_{t}=\sigma\{N(s), s \leq t, x(s), s \leq t+1\} ;
$$

we observe that $\boldsymbol{u}(t) \in \Re_{t}$ and $E\left(\boldsymbol{u}(t) \mid \mathrm{B}_{t-1}\right)=0$. Consequently, $\{\boldsymbol{u}\}$ is a martingale difference (MD) [29, ch. 
VII] process with respect to the family of $\sigma$-fields $\{B\}$. Notationally, we say $\{\boldsymbol{u}\}$ is a $\{囚\}$-MD.

We may define the individual rates $\lambda_{i}(t)$ of the components of $N(t)$ in terms of the elements of $\Lambda(t)$. Since the $2^{m}$ events $S^{\alpha_{1} \cdots \alpha_{m}}=\bigcap_{i=1}^{m} S_{i}^{\alpha_{i}}$ are mutually exclusive, the law of total probability requires that

$$
\begin{aligned}
\lambda_{i}(t) & =P\left\{N_{i}(t)=1 \mid \mathrm{B}_{t-1}\right\}=\sum_{\substack{\alpha_{1} \cdots \alpha_{m} \\
\alpha_{i}=1}} \lambda^{\alpha_{1} \cdots \alpha_{m}}(t) \\
& =\sum_{\substack{\alpha_{1} \cdots \alpha_{m} \\
\alpha_{i}=1}} \sum_{i=1}^{r}\left[\rho_{i}^{\alpha_{1} \cdots \alpha_{m}}(t)\right]^{T} x_{i}(t)
\end{aligned}
$$

where the summation is taken over all $\alpha_{j}$, except for the $i$ th term $\alpha_{i}$, which is set to unity. We may render this expression in matrix notation by defining the vector

is an $r \times m$ matrix. As examples, it is instructive to take cases where $m=2$ and $m=3$. For $m=2$, we obtain, for constant $\boldsymbol{R}$,

thus,

$$
\begin{aligned}
& s_{1}=r^{11}+r^{10} \\
& s_{2}=r^{11}+r^{01}
\end{aligned}
$$

$$
\left[\begin{array}{c}
\lambda_{1}(t) \\
\lambda_{2}(t)
\end{array}\right]=\left[\begin{array}{c}
\rho_{1}^{11}+\rho_{1}^{10} \cdots \rho_{r}^{11}+\rho_{r}^{10} \\
\rho_{1}^{11}+\rho_{1}^{01} \cdots \rho_{r}^{11}+\rho_{r}^{01}
\end{array}\right]\left[\begin{array}{c}
x_{1}(t) \\
\vdots \\
x_{r}(t)
\end{array}\right] .
$$

For $m=3$, we obtain

$$
\begin{aligned}
& s_{1}=r^{111}+r^{110}+r^{101}+r^{100} \\
& s_{2}=r^{111}+r^{110}+r^{011}+r^{010} \\
& s_{3}=r^{111}+r^{101}+r^{011}+r^{001}
\end{aligned}
$$

thus,

$$
\left[\begin{array}{c}
\lambda_{1}(t) \\
\lambda_{2}(t) \\
\lambda_{3}(t)
\end{array}\right]=\left[\begin{array}{c}
\rho_{1}^{111}+\rho_{1}^{110}+\rho_{1}^{101}+\rho_{1}^{100} \cdots \rho_{r}^{111}+\rho_{r}^{110}+\rho_{r}^{101}+\rho_{r}^{100} \\
\rho_{1}^{111}+\rho_{1}^{110}+\rho_{1}^{011}+\rho_{1}^{010} \cdots \rho_{r}^{111}+\rho_{r}^{110}+\rho_{r}^{011}+\rho_{r}^{010} \\
\rho_{1}^{111}+\rho_{1}^{101}+\rho_{1}^{011}+\rho_{1}^{001} \cdots \rho_{r}^{111}+\rho_{r}^{101}+\rho_{r}^{011}+\rho_{r}^{001}
\end{array}\right]\left[\begin{array}{c}
x_{1}(t) \\
\vdots \\
x_{r}(t)
\end{array}\right] \text {. }
$$

$$
\begin{aligned}
& r^{\alpha_{1} \cdots \alpha_{m}}(t)= {\left[\begin{array}{c}
\rho_{1}^{\alpha_{1} \cdots \alpha_{m}}(t) \\
\rho_{2}^{\alpha_{2} \cdots \alpha_{m}}(t) \\
\vdots \\
\rho_{r}^{\alpha_{1} \cdots \alpha_{m}}(t)
\end{array}\right], } \\
& \alpha_{1} \cdots \alpha_{m} \in\left\{\begin{array}{ll}
0 & 1
\end{array}\right\}^{m} .
\end{aligned}
$$

Notice that the $\boldsymbol{r}(t)$ vectors compose the matrix $\boldsymbol{R}(t)$ by columns, whereas the vectors $\boldsymbol{\rho}_{k}^{T}(t)$ compose the matrix $\boldsymbol{R}(t)$ by rows, i.e.,

$$
\boldsymbol{R}(t)=\left[\begin{array}{c}
\boldsymbol{\rho}_{1}^{T}(t) \\
\vdots \\
\boldsymbol{\rho}_{r}^{T}(t)
\end{array}\right]=\left[\boldsymbol{r}^{11 \cdots 11}(t) \cdots \boldsymbol{r}^{00 \cdots 01}(t)\right] .
$$

Thus, we may write

$$
\lambda_{i}(t)=\left\{\sum_{\substack{\alpha_{1} \cdots \alpha_{m} \\ \alpha_{i}=1}} r^{\alpha_{1} \cdots \alpha_{m}}(t)\right\}^{T} \boldsymbol{x}(t)=\boldsymbol{s}_{i}^{T}(t) \boldsymbol{x}(t) .
$$

where

$$
s_{i}(t)=\sum_{\substack{\alpha_{1} \cdots \alpha_{m} \\ \alpha_{i}=1}} \boldsymbol{r}^{\alpha_{1} \cdots \alpha_{m}}(t), \quad i=1, \cdots, m .
$$

Since $\lambda \in[0,1]$ we must have the elements of each $s_{i}$ vector bounded by unity, i.e.,

$$
0 \leq s_{i j} \leq 1, \quad i=1, \cdots, m, j=1, \cdots r .
$$

Consequently, we may express the vector of rates $\lambda(t)=$ $\left[\lambda_{1}(t), \cdots, \lambda_{m}(t)\right]^{T}$ as

where

$$
\lambda(t)=S^{T}(t) x(t)
$$

$$
S(t)=\left[s_{1}(t), s_{2}(t), \cdots, s_{m}(t)\right]
$$

\section{B. Estimation Procedure}

A useful characterization of the point process $\{N\}$ is to obtain its Doob decomposition with respect to $\{\mathbb{B}\}$. The Doob decomposition [30, ch. 2] of a process $\{\boldsymbol{N}\}$ with respect to a family of $\sigma$-fields $\{ß\}$ is the representation

$$
N(t)=\lambda(t)+w(t)
$$

where $\lambda(t)$ is a $\{Q\}$-predictable process (i.e., $\lambda(t) \in$ $\beta_{t-1}$ for all $\left.t\right)$ and $w(t)$ is a $\{\Theta\}$-MD sequence (i.e., $\boldsymbol{w}(t) \in \beta_{t}$ and $\left.E\left(\boldsymbol{w}(t) \mid \Theta_{t-1}\right)=0\right)$. From the above development,

$$
\lambda(t)=E\left(N(t) \mid \wp_{t-1}\right),
$$

and if we define

$$
w(t)=N(t)-E\left(N(t) \mid \bigotimes_{t-1}\right),
$$

then

$$
N(t)=\lambda(t)+w(t)=S^{T}(t) x(t)+w(t)
$$

is the (unique) Doob decomposition of $\{N\}$ with respect to $\{\cap\}$.

Equations (7) and (10) represent a type of state-space model for the system under study. The dynamics equation (7) describes the evolution of the process $\boldsymbol{x}(t)$ over time and is analogous to a linear difference equation driven by a noise process. The observation equation (10) provides the relationship of the observed process $N(t)$ to the state and is analogous to the signal-in-additive-noise process familiar to linear estimation problems.

Although (7) and (10) are linear in all terms, they cannot be treated with standard results from linear estimation theory since the processes $\{\boldsymbol{u}\}$ and $\{\boldsymbol{w}\}$ are not additive white noise (i.e., uncorrelated) processes (in [31] it is 
pointed out that the MD property is an intermediate property between independence and uncorrelation in that independent processes possess the MD property and MD processes possess the uncorrelation property). A key property of the MD processes $\{\boldsymbol{u}\}$ and $\{\boldsymbol{w}\}$ is that $\boldsymbol{u}(t)$ and $\boldsymbol{w}(t)$ are completely unpredictable given $\Theta_{t-1}$. This is the basic property to be exploited in developing the estimator for essentially the same reason that uncorrelation is basic in developing standard linear estimators. Linear estimation techniques, therefore, are not appropriate for this problem since the MD property is more restrictive than the uncorrelation property.

If $\boldsymbol{x}(t)$ were known, the problem of predicting $N(t)$ at any time $t$ would be solved, regardless of the past history of $N(\cdot)$. Unfortunately, $x(t)$ is not directly observable; only $N(t)$ is observed. We are thus faced with the problem of estimating $x(t)$ in order to render an acceptable prediction of $N(t)$. To formulate this estimation problem more clearly, let us define the family of $\sigma$-fields $\mathfrak{F}_{t}$ as

$$
\mathcal{F}_{t}=\sigma\{N(s), s \leq t\}
$$

and compute the conditional expectation of $x(t)$ given $\mathcal{F}_{t-1}$. To do this, we draw upon two fundamental results of martingale theory, namely, the innovations theorem and the representation theorem [31]. Application of these theorems results in a nonlinear estimation procedure to obtain the Doob decomposition of $\{\boldsymbol{N}\}$ with respect to $\{\mathfrak{F}\}$, yielding

$$
\begin{aligned}
N(t) & =\hat{\lambda}(t \mid t-1)+v(t) \\
& =S^{T}(t) \hat{x}(t \mid t-1)+v(t)
\end{aligned}
$$

where $\{\boldsymbol{v}\}$ is an $\{\mathcal{F}\}$-MD process and $\hat{x}(t \mid t-1)$ is the conditional expectation of $\boldsymbol{x}(t)$ given $\mathfrak{F}_{t-1}$.

The process $\boldsymbol{x}(t)$ modulates the rate of the discrete-time vector process $N(t)$ according to (7) and (10). We wish to obtain equations of evolution of the process

$$
\hat{\boldsymbol{x}}(t+1 \mid t)=E\left\{\boldsymbol{x}(t+1) \mid \mathfrak{F}_{t}\right\}=E^{\mathfrak{F}_{t}} \boldsymbol{x}(t+1),
$$

the conditional expectation of $x(t+1)$ given the $\sigma$-field $\mathcal{F}_{t}$. We follow the results of [32] and obtain an estimator of the form

$$
\begin{aligned}
\hat{\boldsymbol{x}}(t+1 \mid t)= & E^{\mathfrak{F}_{t-1}} \boldsymbol{x}(t+1) \\
& +(\boldsymbol{\mu}, \boldsymbol{v})_{t}(\boldsymbol{v}, \boldsymbol{v})_{t}^{-1} \boldsymbol{v}(t)
\end{aligned}
$$

where

$$
\boldsymbol{\mu}(t)=E^{\mathcal{T}_{t}} \boldsymbol{x}(t+1)-E^{\mathcal{F}_{t-1}} \boldsymbol{x}(t+1)
$$

and

$$
\begin{aligned}
v(t) & =N(t)-E^{\mathcal{F}_{t-1}} N(t) \\
& =N(t)-S^{T}(t) E^{\mathcal{F}_{t-1}} x(t),
\end{aligned}
$$

the matrix

$$
(\boldsymbol{\mu}, \boldsymbol{v})_{t}=E^{\mathcal{F}_{t-1}} \boldsymbol{\mu}(t) \boldsymbol{v}^{T}(t)
$$

is the conditional covariance of $\mu(t)$ and $\boldsymbol{v}(t)$, and the matrix

$$
(\boldsymbol{v}, \boldsymbol{v})_{t}=E^{\mathfrak{F}_{t-1}} \mathbf{v}(t) \mathbf{v}^{T}(t)
$$

is the conditional variance of $v(t)$.

The conditional covariance $(\mu, v)_{t}$ (see [32]) is the $r$ $\times m$ matrix

$$
\begin{aligned}
E^{\mathcal{F}_{t-1}} & {\left[\boldsymbol{\mu}(t) \mathbf{v}^{T}(t)\right] } \\
= & \boldsymbol{Q}^{T}(t) \operatorname{diag}\{\hat{\boldsymbol{x}}(t \mid t-1)\} S(t) \\
& -\boldsymbol{Q}^{T}(t) \hat{\boldsymbol{x}}(t \mid t-1) \hat{\boldsymbol{x}}^{T}(t \mid t-1) S(t) \\
= & \boldsymbol{Q}^{T}(t)[\operatorname{diag}\{\hat{\boldsymbol{x}}(t \mid t-1)\} \\
& \left.-\hat{\boldsymbol{x}}(t \mid t-1) \hat{\boldsymbol{x}}^{T}(t \mid t-1)\right] \boldsymbol{S}(t)
\end{aligned}
$$

where diag $\{\cdot\}$ denotes a diagonal matrix whose diagonal elements are composed of the elements of the vector argument.

The conditional variance $(v, v)_{t}[32]$ is the $m \times m$ matrix

$$
\begin{aligned}
& E^{\mathcal{F}_{t-1}}\left[\boldsymbol{v}(t) \boldsymbol{v}^{T}(t)\right] \\
& =\left[\begin{array}{cccc}
\hat{\lambda}_{1}-\left(\hat{\lambda}_{1}\right)^{2} & \hat{\lambda}_{12}-\hat{\lambda}_{1} \hat{\lambda}_{2} & \cdots & \hat{\lambda}_{1 m}-\hat{\lambda}_{1} \hat{\lambda}_{m} \\
\hat{\lambda}_{12}-\hat{\lambda}_{1} \hat{\lambda}_{2} & \hat{\lambda}_{2}-\left(\hat{\lambda}_{2}\right)^{2} & \cdots & \hat{\lambda}_{2 m}-\lambda_{2} \hat{\lambda}_{m} \\
\vdots & \vdots & \vdots \vdots & \vdots \\
\hat{\lambda}_{1 m}-\hat{\lambda}_{1} \hat{\lambda}_{m} & \hat{\lambda}_{2 m}-\hat{\lambda}_{2} \hat{\lambda}_{m} & \cdots & \hat{\lambda}_{m}-\left(\hat{\lambda}_{m}\right)^{2}
\end{array}\right]
\end{aligned}
$$

where the time arguments $(t \mid t-1)$ on $\hat{\lambda}$ have been suppressed for brevity, with

$$
\hat{\lambda}_{i}(t \mid t-1)=s_{i}^{T}(t) \hat{x}(t \mid t-1)
$$

and

$$
\begin{aligned}
\hat{\boldsymbol{\lambda}}_{i j}(t \mid t-1) & =\sum_{\substack{\alpha_{1} \cdots \alpha_{m} \\
\alpha_{i}=\alpha_{j}=1}} \hat{\lambda}^{\alpha_{1} \cdots \alpha_{m}}(t \mid t-1) \\
& =\sum_{\substack{\alpha_{1} \cdots \alpha_{m} \\
\alpha_{i}=\alpha_{j}=1}}\left(\boldsymbol{\rho}^{\alpha_{1} \cdots \alpha_{m}}\right)^{T}(t) \hat{\boldsymbol{x}}(t \mid t-1),
\end{aligned}
$$

i.e., the sum is taken over all $\alpha_{1} \cdots \alpha_{m}$ such that $\alpha_{i}=$ $\alpha_{j}=1$. Define

$$
\boldsymbol{s}_{i j}(t)=\sum_{\substack{\alpha_{1} \cdots \alpha_{m} \\ \alpha_{i}=\alpha_{j}=1}} \boldsymbol{\rho}^{\alpha_{1} \cdots \alpha_{m}}(t)
$$

to obtain

$$
\hat{\lambda}_{i j}(t \mid t-1)=s_{i j}^{T}(t) \hat{x}(t \mid t-1) .
$$

Thus, the estimator becomes, using (18) and (19),

$$
\begin{aligned}
\hat{\boldsymbol{x}}(t+1 \mid t)= & \boldsymbol{Q}^{T}(t) \hat{\boldsymbol{x}}(t \mid t-1)+E^{\mathcal{F}_{t-1}}\left[\boldsymbol{\mu}(t) \boldsymbol{v}^{T}(t)\right] \\
& \cdot\left[E^{\mathscr{F}_{t-1}}\left[\boldsymbol{v}(t) \boldsymbol{v}^{T}(t)\right]\right]^{-1} \boldsymbol{v}(t) .
\end{aligned}
$$

This estimator is recursive, which provides the capability of real-time implementation. It is necessary, however, to 
initialize this estimator with

$$
\hat{\boldsymbol{x}}(1 \mid 0)=x_{0}
$$

where $x_{0}$ is given. Typically, this quantity would reflect the uniform distribution or other a priori information available at time $t=0$. The resulting rate estimate for the rate vector is, therefore,

$$
\hat{\boldsymbol{\Lambda}}(t+1 \mid t)=\boldsymbol{R}^{T}(t+1) \hat{\boldsymbol{x}}(t+1 \mid t) .
$$

1) Examples of Covariances As examples of $(v, v)_{t}$ consider the cases $m=2$ and $m=3$. For $m=2$, we have only one term in the summation for $\hat{\lambda}_{10}(t \mid t-1)=$ $\hat{\lambda}_{01}(t \mid t-1)$, and the convariance matrix is

$$
E^{\mathcal{F}_{t-1}}\left[v(t) v^{T}(t)\right]=\left[\begin{array}{ll}
\hat{\lambda}_{1}-\left(\hat{\lambda}_{1}\right)^{2} & \hat{\lambda}_{12}-\hat{\lambda}_{1} \hat{\lambda}_{2} \\
\hat{\lambda}_{12}-\hat{\lambda}_{1} \hat{\lambda}_{2} & \hat{\lambda}_{2}-\left(\hat{\lambda}_{2}\right)^{2}
\end{array}\right]
$$

(the time argument has been dropped for brevity) where, following (20),

$$
\begin{aligned}
& \hat{\lambda}_{1}=\hat{\lambda}^{11}+\hat{\lambda}^{10} \\
& \hat{\lambda}_{2}=\hat{\lambda}^{11}+\hat{\lambda}^{01}
\end{aligned}
$$

and, following (21),

$$
\hat{\lambda}_{12}=\hat{\lambda}^{11}
$$

For $m=3$, we have

$$
\begin{aligned}
E^{\mathcal{F}_{t-1}}\left[\mathbf{v}(t) \mathbf{v}^{T}(t)\right] & \\
= & {\left[\begin{array}{lll}
\hat{\lambda}_{1}-\left(\hat{\lambda}_{1}\right)^{2} & \hat{\lambda}_{12}-\hat{\lambda}_{1} \hat{\lambda}_{2} & \hat{\lambda}_{13}-\hat{\lambda}_{1} \hat{\lambda}_{3} \\
\hat{\lambda}_{12}-\hat{\lambda}_{1} \hat{\lambda}_{2} & \hat{\lambda}_{2}-\left(\hat{\lambda}_{2}\right)^{2} & \hat{\lambda}_{23}-\hat{\lambda}_{2} \hat{\lambda}_{3} \\
\hat{\lambda}_{13}-\hat{\lambda}_{1} \hat{\lambda}_{3} & \hat{\lambda}_{23}-\hat{\lambda}_{2} \hat{\lambda}_{3} & \hat{\lambda}_{3}-\left(\hat{\lambda}_{3}\right)^{2}
\end{array}\right] }
\end{aligned}
$$

where

$$
\begin{aligned}
& \hat{\lambda}_{1}=\hat{\lambda}^{111}+\hat{\lambda}^{110}+\hat{\lambda}^{101}+\hat{\lambda}^{100} \\
& \hat{\lambda}_{2}=\hat{\lambda}^{111}+\hat{\lambda}^{110}+\hat{\lambda}^{011}+\hat{\lambda}^{010} \\
& \hat{\lambda}_{3}=\hat{\lambda}^{111}+\hat{\lambda}^{101}+\hat{\lambda}^{011}+\hat{\lambda}^{001}
\end{aligned}
$$

and

$$
\begin{aligned}
& \hat{\lambda}_{12}=\hat{\lambda}^{111}+\hat{\lambda}^{110} \\
& \hat{\lambda}_{13}=\hat{\lambda}^{111}+\hat{\lambda}^{101} \\
& \hat{\lambda}_{23}=\hat{\lambda}^{111}+\hat{\lambda}^{011} .
\end{aligned}
$$

\section{Covariance of Estimation Error}

The recursive estimator given by (22) and the corresponding rate estimate given by (23) represent the minimum mean-square error prediction of $x(t+1)$ and $\boldsymbol{\Lambda}(t+1)$ given $\mathfrak{F}_{t}$, the past and present data. We may obtain the conditional uncertainty on these estimates by computing the conditional covariance matrix of the estimation error

$$
\begin{aligned}
\tilde{\boldsymbol{x}}(t+1 \mid t) & =\boldsymbol{x}(t+1)-\hat{\boldsymbol{x}}(t+1 \mid t) \\
\tilde{\boldsymbol{\Lambda}}(t+1 \mid t) & =\boldsymbol{\Lambda}(t+1)-\hat{\boldsymbol{\Lambda}}(t+1 \mid t) .
\end{aligned}
$$

The estimation error covariance of $\tilde{\boldsymbol{x}}(t+1 \mid t)$ may be computed as

$$
\begin{aligned}
P(t+1 \mid t)= & E^{\mathcal{f}_{t}} \tilde{\boldsymbol{x}}(t+1 \mid t) \tilde{\boldsymbol{x}}^{T}(t+1 \mid t) \\
= & E^{\mathcal{F}_{t}} \boldsymbol{x}(t+1) \boldsymbol{x}^{T}(t+1) \\
& -\hat{\boldsymbol{x}}(t+1 \mid t) \hat{\boldsymbol{x}}^{T}(t+1 \mid t) \\
= & E^{\mathcal{F}_{t}} \operatorname{diag}\{\boldsymbol{x}(t+1)\} \\
& -\hat{\boldsymbol{x}}(t+1 \mid t) \hat{\boldsymbol{x}}^{T}(t+1 \mid t) \\
= & \operatorname{diag}\{\hat{\boldsymbol{x}}(t+1 \mid t)\} \\
& -\hat{\boldsymbol{x}}(t+1 \mid t) \hat{\boldsymbol{x}}^{T}(t+1 \mid t),
\end{aligned}
$$

and by (23), we have the covariance of the rate estimation error given by

$$
\begin{aligned}
\Pi(t+1 \mid t)= & E^{\mathcal{F}_{t}} \tilde{\boldsymbol{\Lambda}}(t+1 \mid t) \tilde{\boldsymbol{\Lambda}}^{T}(t+1 \mid t) \\
= & \boldsymbol{S}^{T}(t+1)[\operatorname{diag}\{\hat{\boldsymbol{x}}(t+1 \mid t)\} \\
& \left.-\hat{\boldsymbol{x}}(t+1 \mid t) \hat{\boldsymbol{x}}^{T}(t+1 \mid t)\right] \boldsymbol{S}(t+1) .
\end{aligned}
$$

At this point, some comments with regard to the recursive estimator provided in (22) may be appropriate. Note that although this estimate is recursive and possesses structure much like a Kalman filter, it is not a linear estimator since the gain matrix is dependent upon the state and, hence, upon the data. Furthermore, the covariance associated with this estimate is a conditional covariance, rather than an unconditional covariance as with the linear case. Note also that this covariance does not obey a Riccati equation, but it is true that the (state-dependent) gain of the estimator is proportional to this covariance, as is the case with the Kalman filter. Although the estimation error covariance provided above is conditional, it may properly be used in practice to assess the quality of the estimate for $\boldsymbol{x}(t+1)$ or $\boldsymbol{\Lambda}(t+1)$.

\section{Decision-Directed Detection}

The estimator defined in (22) provides an estimate of the vector $\boldsymbol{x}(t)$, thus yielding a probability vector $\hat{\boldsymbol{x}}(t \mid t-1)$ with components $\hat{x}_{i}(t \mid t-1)$ representing the conditional probability that $\boldsymbol{\Lambda}(t)=\boldsymbol{\rho}_{i}(t)$. The conditional expectation of $\boldsymbol{\Lambda}(t)$ given the $\sigma$-field $\mathfrak{F}_{t-1}$ is

$$
\hat{\boldsymbol{\Lambda}}(t \mid t-1)=\boldsymbol{R}^{T}(t) \hat{\boldsymbol{x}}(t \mid t-1),
$$

which yields rate estimates $\hat{\lambda}^{\alpha_{1} \cdots \alpha_{m}}(t \mid t-1)$, with $\hat{\lambda}^{0 \cdots 0}(t \mid t-1)=1-\Sigma_{\alpha_{1} \cdots \alpha_{m}} \hat{\lambda}^{\alpha_{1} \cdots \alpha_{m}}(t \mid t-1)$. The joint distribution of $N(t)$ is, therefore, given by

$$
p_{N(t)}\left(\alpha_{1} \cdots \alpha_{m} \mid F_{t-1}\right)=\hat{\lambda}^{\alpha_{1} \cdots \alpha_{m}}(t \mid t-1) .
$$

\section{A. Detector Structure}

Let $f\left(\boldsymbol{y}(t) \mid \mathcal{S}^{\alpha_{1} \cdots \alpha_{m}}\right)$ denote the joint distribution of $\boldsymbol{y}(t)$ under the hypothesis that $S^{\alpha_{1} \cdots \alpha_{m}}$ occurs for $\alpha_{1} \cdots$ $\alpha_{m} \in\{0,1\}^{m}$. Under the decision-directed strategy, the estimator given by (25) may be used to characterize the prior distribution $\pi^{\alpha_{1} \cdots \alpha_{m}}(t)$ given by (3) to be used to calculate the likelihood function given in (2). Since the events $D_{i}[y(t)]$ defined by (1) are not mutually exclu- 
sive, the decision-directed empirical Bayes rule for the $M$ $=2^{m}$ events $S^{\alpha_{1} \cdots \alpha_{m}}$ assumes the form

$$
\begin{aligned}
& \delta\left(\mathcal{S}^{\beta_{1} \cdots \beta_{m}} \mid \boldsymbol{y}\right) \\
& 1 \text { if } \hat{\pi}^{\beta_{1} \cdots \beta_{m}} f\left(y \mid \delta^{\beta_{1} \cdots \beta_{m}}\right) \\
& =\left\{\quad=\max _{\alpha_{1}, \cdots, \alpha_{m}}\left\{\hat{\pi}^{\alpha_{1} \cdots \alpha_{m}} f\left(\boldsymbol{y} \mid \mathcal{S}^{\alpha_{1} \cdots \alpha_{m}}\right)\right\}\right. \\
& 0 \text { otherwise. }
\end{aligned}
$$

Fig. 2 represents a geometrical interpretation of this decision rule. Let $\boldsymbol{J}$ denote a partitioning of $\boldsymbol{Y}$ into $M=2^{m}$ disjoint subsets $\left\{T^{\alpha_{1} \cdots \alpha_{m}}\right\}, \alpha_{1} \cdots \alpha_{m} \in\{0,1\}^{m}$, according to the rule

$$
\delta\left(\mathcal{S}^{\alpha_{1} \cdots \alpha_{m}} \mid \boldsymbol{y}(t)\right)=1 \Leftrightarrow \boldsymbol{y}(t) \in T^{\alpha_{1} \cdots \alpha_{m}} .
$$

Thus, the decision rule may be implemented by testing the received signal $\boldsymbol{y}(t)$ to determine in which of the sets $T^{\alpha_{1} \cdots \alpha_{m}}$ it resides. The elements of $\mathfrak{J}$ are determined by the distribution function $f\left(\boldsymbol{y} \mid S^{\alpha_{1} \cdots \alpha_{m}}\right)$ and the estimate for the prior, $\hat{\pi}^{\alpha_{1} \cdots \alpha_{m}}$.

The simplest form of the estimator for $\pi^{\alpha_{1} \cdots \alpha_{m}}(t)$ is to set

$$
\hat{\pi}^{\alpha_{1} \cdots \alpha_{m}}(t)=\hat{\lambda}^{\alpha_{1} \cdots \alpha_{m}}(t \mid t-1)
$$

which incorporates the assumption that $\hat{\lambda}$ is an unbiased estimator of $\pi$. Using this rule, the decision function takes a simple form. Based on the previous outputs $N(s), s \leq$ $t-1$, the rate estimator yields estimates $\hat{\lambda}^{\alpha_{1}} \cdots \alpha_{m}$ $(t \mid t-1)$, which may be incorporated into a generalized likelihood ratio test of the form

$$
\begin{aligned}
& \delta\left(\mathcal{S}^{\beta_{1} \cdots \beta_{m}} \mid \boldsymbol{y}\right) \\
& \quad= \begin{cases}1 & \text { if } \hat{\lambda}^{\beta_{1} \cdots \beta_{m}} f\left(\boldsymbol{y} \mid \mathcal{S}^{\beta_{1} \cdots \beta_{m}}\right) \\
& =\max _{\alpha_{1}, \cdots, \alpha_{m}}\left\{\hat{\boldsymbol{\lambda}}^{\alpha_{1} \cdots \alpha_{m}} f\left(\boldsymbol{y} \mid \mathcal{S}^{\alpha_{1} \cdots \alpha_{m}}\right)\right\} \\
0 & \text { otherwise. }\end{cases}
\end{aligned}
$$

Equation (28) means that the hypothesis $H_{\beta_{1}} \cdots \beta_{m}$ is accepted at time $t$. Thus, the vector $N(t)$ is

$$
N(t)=\left[\begin{array}{c}
\beta_{1} \\
\beta_{2} \\
\vdots \\
\beta_{m}
\end{array}\right] .
$$

This vector is then used to update the estimate for $\mathbf{\Lambda}(t)$, yielding $\hat{\boldsymbol{\Lambda}}(t \mid t-1)$, which will be used to adjust the prior probabilities in the generalized likelihood ratio test for $t+1$ in a recursive manner, as illustrated in Fig. 3 .

\section{B. Bias Correction}

Unfortunately, it is not generally true that $\hat{\lambda}$ is an unbiased estimate of $\pi$, and we must investigate the effects

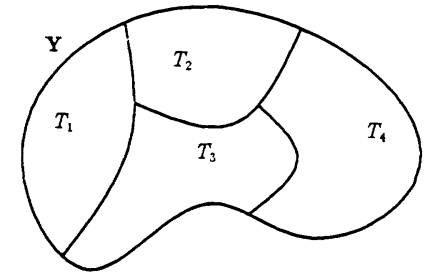

Fig. 2. Geometrical interpretation of decision rule (for $m=2$ ).

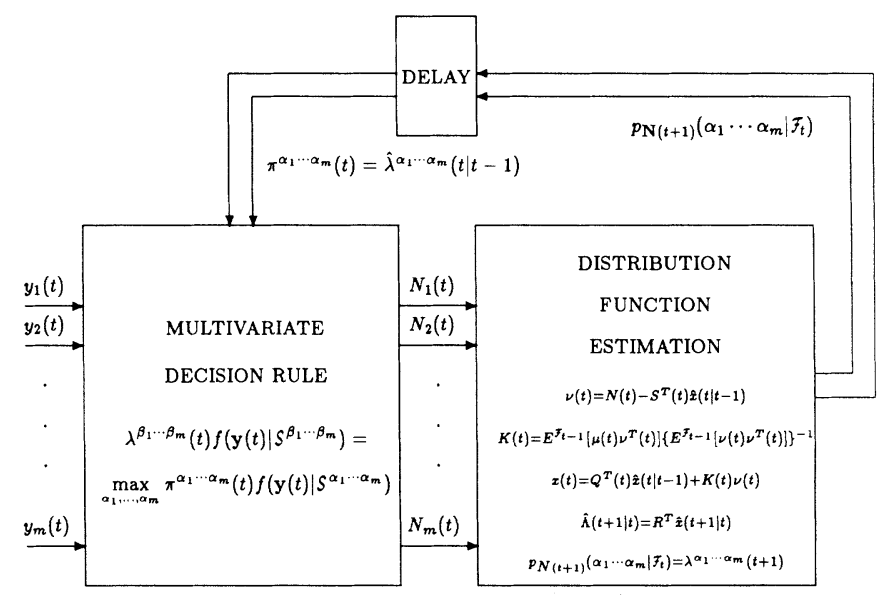

Fig. 3. Decision-directed detector.

of this bias on the performance of the detector and, if necessary, explore methods of eliminating or reducing this bias.

For any partitioning $J$, we may express $\lambda^{\alpha_{1} \cdots \alpha_{m}}(t)$ in terms of $\pi^{\alpha_{1} \cdots \alpha_{m}}(t)$ as

$$
\begin{aligned}
& \lambda^{\alpha_{1} \cdots \alpha_{m}}(t)=\pi^{\alpha_{1} \cdots \alpha_{m}}(t) \\
\cdot & \int_{T^{\alpha_{1} \cdots \alpha_{m}}}\left[f\left(\boldsymbol{y} \mid \mathcal{S}^{\alpha_{1} \cdots \alpha_{m}}\right)-f\left(\boldsymbol{y} \mid \bar{S}^{\alpha_{1} \cdots \alpha_{m}}\right)\right] d \boldsymbol{y} \\
+ & \int_{T^{\alpha_{1} \cdots \alpha_{m}}} f\left(\boldsymbol{y} \mid \overline{\boldsymbol{S}}^{\alpha_{1} \cdots \alpha_{m}}\right) d \boldsymbol{y},
\end{aligned}
$$

and solving for $\pi^{\alpha_{1} \cdots \alpha_{m}}(t)$ yields

$$
\begin{aligned}
\pi^{\alpha_{1} \cdots \alpha_{m}}(t) & \frac{\lambda^{\alpha_{1} \cdots \alpha_{m}}(t)-\int_{T^{\alpha_{1} \cdots \alpha_{m}}} f\left(\boldsymbol{y} \mid \overline{\mathcal{S}}^{\alpha_{1} \cdots \alpha_{m}}\right) d \boldsymbol{y}}{\int_{T^{\alpha_{1} \cdots \alpha_{m}}}\left[f\left(\boldsymbol{y} \mid \mathcal{S}^{\alpha_{1} \cdots \alpha_{m}}\right)-f\left(\boldsymbol{y} \mid \overline{\mathcal{S}}^{\alpha_{1} \cdots \alpha_{m}}\right)\right] d \boldsymbol{y}} \\
= & a\left(T^{\alpha_{1} \cdots \alpha_{m}}\right) \lambda^{\alpha_{1} \cdots \alpha_{m}}(t)+b\left(T^{\alpha_{1} \cdots \alpha_{m}}\right)
\end{aligned}
$$

where $a(\cdot)$ and $b(\cdot)$ are defined in an obvious way. Thus, the true rate $\pi$ is expressed as a linear function of the detected rate $\lambda$. In general, $a(\cdot) \neq 1$ and $b(\cdot) \neq 0$. However, for any given decision region $T^{\alpha_{1} \cdots \alpha_{m}}$, the correction terms may be computed and applied. If we estimate $\lambda^{\alpha_{1} \cdots \alpha_{m}}(t)$ using the above scheme, we may then express the estimate of $\pi^{\alpha_{1} \cdots \alpha_{m}}(t)$ as

$$
\begin{aligned}
\hat{\pi}^{\alpha_{1} \cdots \alpha_{m}}(t)= & a\left(T^{\alpha_{1} \cdots \alpha_{m}}\right) \hat{\lambda}^{\alpha_{1} \cdots \alpha_{m}}(t \mid t-1) \\
& +b\left(T^{\alpha_{1} \cdots \alpha_{m}}\right) .
\end{aligned}
$$


This structure holds for all values of $T^{\alpha_{1} \cdots \alpha_{m}}$ and, in particular, holds when the partition regions $T^{\alpha_{1} \cdots \alpha_{m}}$ are specified by the previous best estimate of the prior, namely, $\hat{\pi}(t-1)$.

There are a number of issues to be considered concerning the removal of the bias. First, it is evident from the structure of (29) that $a(\cdot) \geq 1$, and therefore, the variance on the estimation error for $\pi^{\alpha_{1} \cdots \alpha_{m}}$ will be greater than the variance on the estimation error for $\lambda^{\alpha_{1} \cdots \alpha_{m}}$; thus, bias may be removed only at the expense of increased uncertainty in the estimate. Second, the integrations indicated in (29) are extremely complex since the integrations are taken over $m$-dimensional space. For the Gaussian case, these integrals cannot be evaluated in closed form, and the computational burden to evaluate them numerically is considerable. For purposes of this analysis, therefore, the bias is not removed, and the estimator defined by (27) is used. In Section IV, Monte Carlo results are presented to provide partial justification for this simplifying procedure.

\section{Monte Carlo Performance Analysis}

\section{A. Signal Model}

A key analysis issue is to assess the performance of the proposed algorithm. The interaction between detection and estimation, however, makes performance analysis of this decision-directed procedure extremely complex. The difficulty is due primarily to the dependences present in the adaptive detector that are introduced by the two-way coupling between detection and estimation, which are virtually impossible to treat since the multivariate distributions are not available in analytic form. Consequently, theoretical performance analysis is not possible. An alternative to a classical performance analysis is to conduct Monte Carlo analyses and to evaluate the performance of this algorithm on the basis of first and second sample moments of the trial results. To this end, we present simulation results to demonstrate the operation of the algorithms presented in Sections II and III and to provide a simplified collection scenario to illustrate the performance of the multivariate decision-directed detection procedure.

We assume the signal is a function $\boldsymbol{\eta}(t)$ embedded in Gaussian noise, i.e.,

$$
\boldsymbol{y}(t)=\boldsymbol{\eta}(t)+\boldsymbol{v}(t)
$$

is a multivariate normal random vector, $\boldsymbol{y}(t) \sim \mathfrak{N}(\boldsymbol{\eta}(t)$, $\boldsymbol{\Sigma})$. For ease of simulation, we will assume that $\boldsymbol{\Sigma}=\sigma^{2} \boldsymbol{I}$ and hence that the elements $y_{i}(t)$ are uncorrelated with variance $\sigma^{2}$.

The data are simulated as follows. If the signal is in class $S_{i}$, the $i$ th element of $\boldsymbol{\eta}(t)$ is $b_{i}(t)$, the mean value of the signal. Otherwise, the $i$ th element of $\boldsymbol{\eta}(t)$ is set to zero. We may then express the mean vector as

$$
\boldsymbol{\eta}(t)=\boldsymbol{B}(t) \boldsymbol{n}(t)
$$

where $\boldsymbol{B}(t)=\operatorname{diag}\left\{b_{1}(t), \cdots, b_{m}(t)\right\}$ and $\boldsymbol{n}(t)=$

$$
\begin{aligned}
& {\left[n_{1}(t), \cdots, n_{m}(t)\right]^{T} \text { where }} \\
& n_{i}(t)= \begin{cases}1 & \text { if signal is in class } S_{i} \\
0 & \text { otherwise. }\end{cases}
\end{aligned}
$$

The vector discrete-time point process $\{\boldsymbol{n}\}$ represents the occurrences of the various classifications at each time $t=$ $0,1, \cdots$. The distribution of this process is given by

$$
P\{\boldsymbol{n}(t)=\boldsymbol{\alpha}\}=\pi^{\alpha_{1} \cdots \alpha_{m}}(t) .
$$

This distribution may be either time varying or constant, but is assumed to be unknown. For purposes of this simulation, $\pi$ will be taken as a constant, and the process $\{\boldsymbol{n}\}$ will be taken as a Bernoulli process. Note that this structure is not required for the successful application of the algorithms, but it represents perhaps the simplest condition to simulate. In general, $\{\boldsymbol{n}\}$ may be a multivariate marked point process.

We consider here the performance of the algorithm for $m=4$. The decision-directed estimator will yield estimates of

$$
\lambda^{\alpha_{1} \alpha_{2} \alpha_{3} \alpha_{4}}=P\left\{S^{\alpha_{1} \alpha_{2} \alpha_{3} \alpha_{4}} \mid \wp_{t-1}\right\}
$$

where

$$
\Re_{t-1}=\sigma\{N(s), s \leq t\}
$$

and where $S^{\alpha_{i}}$ occurs for $\alpha_{i}=1$ and $\alpha_{i}=0$ denotes classifying $y_{i}(t)$ as arising from the signal-plus-noise or noise-only situations, respectively.

\section{B. Markov Chain Model}

As the dimension $m$ is increased, the number of possible states in the vector Markov chain tends to grow exponentially. The dimensionality of the problem may be greatly reduced if the joint probability possesses a special structure that may be exploited. In this study, we make the assumption that the density function for $\boldsymbol{y}(t)$ factors into the product of $k$ marginal densities for each $t$ as follows (dropping the time argument for brevity):

$$
\begin{aligned}
f\left(y_{1}, \cdots, y_{m}\right)= & f\left(y_{1} \mid y_{2}\right) f\left(y_{2} \mid y_{3}\right) \\
& \cdots f\left(y_{m-1} \mid y_{m}\right) f\left(y_{m} \mid y_{1}\right) .
\end{aligned}
$$

This factorization is motivated by the reasonable condition that the distribution of each component of the signal depends only upon its immediate neighbors and has application in a surveillance scenario wherein signals are to be detected in azimuthal sectors [33]. With this assumption, it is possible to deal with four 2-dimensional problems rather than one 16-dimensional problem, as follows. We may express the joint distribution of $N(t)$ (for $m=$ 4) as

$$
\begin{aligned}
& p\left(N_{1}, N_{2}, N_{3}, N_{4}\right) \\
& \quad=\frac{p\left(N_{1}, N_{2}\right) p\left(N_{2}, N_{3}\right) p\left(N_{3}, N_{4}\right) p\left(N_{4}, N_{1}\right)}{p\left(N_{2}\right) p\left(N_{3}\right) p\left(N_{4}\right) p\left(N_{1}\right)} .
\end{aligned}
$$


Using this factorization, we need estimate only the four quantities $p\left(N_{i}, N_{i+1}\right)$ where the indexes are taken modulo 4 . The results must then be combined as in (30) to yield the joint distribution.

For the resulting two-dimensional estimation problem, the values of $\boldsymbol{R}$ and $\boldsymbol{Q}$ are as follows:

$$
\begin{aligned}
\boldsymbol{R}^{T} & =\left[\begin{array}{llllll}
0.05 & 0.05 & 0.475 & 0.475 & 0.05 & 0.90 \\
0.05 & 0.475 & 0.05 & 0.475 & 0.90 & 0.05
\end{array}\right] \\
\boldsymbol{Q}^{T} & =\left[\begin{array}{llllll}
0.90 & 0.04 & 0.04 & 0.00 & 0.02 & 0.02 \\
0.04 & 0.90 & 0.01 & 0.15 & 0.04 & 0.00 \\
0.04 & 0.01 & 0.90 & 0.15 & 0.00 & 0.04 \\
0.00 & 0.01 & 0.01 & 0.40 & 0.04 & 0.04 \\
0.01 & 0.04 & 0.00 & 0.15 & 0.90 & 0.00 \\
0.01 & 0.00 & 0.04 & 0.15 & 0.00 & 0.90
\end{array}\right]
\end{aligned}
$$

The columns of $\boldsymbol{R}^{T}$ are the states of the vector Markov chain and represent the states to which the rate vector $\boldsymbol{\Lambda}$ may transit as time evolves. The values assumed by this matrix and the dimension $r=6$ are not nearly as restrictive as they may appear, since the estimate $\hat{\boldsymbol{x}}$ will in general be a convex linear combination of all these states and the rate estimate $\hat{\boldsymbol{\Lambda}}$ will range continuously over the convex closure of the vectors $\boldsymbol{\rho}_{1}, \cdots, \boldsymbol{\rho}_{r}$. Since the estimated rate must lie in this convex closure, runaway may be eliminated by bounding the closure away from degenerate values (i.e., where all of the priors are zero except one). Runaway occurs when the decision-directed detector diverges to a degenerate probability distribution. In [22], it is shown that the probability of runaway is exponentially small for the binary case with stationary priors. For the multivariate nonstationary case, no analytical results have been obtained, but simulation results indicate that runaway is not a problem with the finite-state Markov chain model.

An element $q_{i j}$ of $\boldsymbol{Q}^{T}$ represents the probability of transiting to state $j$ of the vector Markov chain at time $t+1$ given that the state is $i$ at time $t$. Note that the strong diagonal structure of $\boldsymbol{Q}^{T}$ indicates that, given the Markov chain is in state $i$ at time $t$, it will likely remain there at time $t+1$. The fact that $q_{44}$ is smaller than the other diagonal elements indicates that the state $\boldsymbol{\rho}_{4}^{T}=[0.475$, 0.475 ] is a transitory one, which is a reasonable assumption.

\section{Estimation of Signal Strength}

We may not assume that the signal strength $b_{i}$ is known a priori, and procedures must be developed to make an estimate $b_{i}$. Probably the most straightforward method, and one that has previously been used with success in [10], [26], is to compute $\hat{b}_{i}$ as the empirical average of those samples $y_{i}(t)$ for which a detection in sector $i$ occurred (i.e., when $S_{i}$ occurs). This estimator assumes the general form

$$
\begin{array}{r}
\hat{b_{i}}(t)=\hat{b}_{i}(t-1)+\frac{N_{i}(t)}{\sum_{s=1}^{t} \gamma^{t-s} N_{s}} \\
\cdot\left[y_{i}(t)-\hat{b}_{i}(t-1)\right]
\end{array}
$$

where the $\gamma$ is a constant such that $0<\gamma \leq 1$. This quantity represents a "forgetting factor," which permits earlier estimates to be discounted in favor of more recent data. Using such a model, smooth changes in $b_{i}(t)$ may be tracked.

The effect of this estimate on the detection procedure is to substitute an estimate $\hat{\boldsymbol{B}}(t)$ for $\boldsymbol{B}(t)$ in the distribution used in (28) to obtain

$$
\begin{aligned}
& \delta\left(\mathcal{S}^{\beta_{1} \cdots \beta_{m}} \mid \boldsymbol{y}\right) \\
& = \begin{cases}1 & \text { if } \hat{\lambda}^{\beta_{1} \cdots \beta_{m}} f\left(\boldsymbol{y} \mid \mathcal{S}^{\beta_{1} \cdots \beta_{m}}, \hat{\boldsymbol{B}}\right) \\
& =\max _{\substack{\alpha_{1}, \cdots, \alpha_{m} \\
\text { otherwise. }}}\left\{\hat{\lambda}^{\alpha_{1} \cdots \alpha_{m}} f\left(\boldsymbol{y} \mid \mathrm{S}^{\alpha_{1} \cdots \alpha_{m}}, \hat{\boldsymbol{B}}\right)\right\}\end{cases}
\end{aligned}
$$

where

$$
\begin{gathered}
f\left(\boldsymbol{y} \mid \mathfrak{S}^{\alpha_{1} \cdots \alpha_{m}}, \hat{\boldsymbol{B}}\right)=|2 \pi \boldsymbol{\Sigma}|^{-1 / 2} \exp \left\{-\frac{1}{2}\left(\boldsymbol{y}-\hat{\boldsymbol{\eta}}^{\alpha_{1} \cdots \alpha_{m}}\right)^{T}\right. \\
\left.\cdot \boldsymbol{\Sigma}^{-1}\left(\boldsymbol{y}-\hat{\boldsymbol{\eta}}^{\alpha_{1} \cdots \alpha_{m}}\right)\right\}
\end{gathered}
$$

is the multivariate normal distribution evaluated at the estimate

$$
\hat{\boldsymbol{\eta}}(t)=\hat{\boldsymbol{B}}(t) \boldsymbol{n}(t)
$$

where $\hat{\boldsymbol{B}}(t)=\operatorname{diag}\left\{\left(\hat{b}_{1}(t-1), \cdots, \hat{b}_{m}(t-1)\right\}\right.$ and $\boldsymbol{n}(t)=\left[\alpha_{1} \cdots \alpha_{m}\right]^{t}$. Note that both the recursion (31) and the decision rule (32) must be initialized with some $a$ priori estimate $\hat{\boldsymbol{B}}(0)$. Furthermore, the ratio

$$
\frac{N_{i}(t)}{\sum_{s=1}^{t} \gamma^{t-s} N_{s}}
$$

must be initialized to zero at $t=0$ to ensure that the estimate for $b_{i}$ is well defined and is equal to the a priori value until observations are obtained.

\section{Simulation Results}

We present selected simulation results to illustrate the performance of the proposed algorithm. If we let $L$ be the number of simulations performed, the sample mean, sample variance, and mean-square estimation error may be computed, respectively, as follows:

$$
\begin{aligned}
\overline{\hat{\lambda}}(t) & =\frac{1}{L} \sum_{i=1}^{L} \hat{\lambda}(t, i) \\
\bar{\sigma}_{\hat{\lambda}}^{2}(t) & =\frac{1}{L} \sum_{i=1}^{L}[\hat{\lambda}(t, i)-\overline{\hat{\lambda}}(t)]^{2} \\
\bar{\epsilon}_{\hat{\lambda}}(\mathrm{t}) & =\frac{1}{L} \sum_{i=1}^{L}[\hat{\lambda}(t, i)-\lambda(t)]^{2}
\end{aligned}
$$


(a)

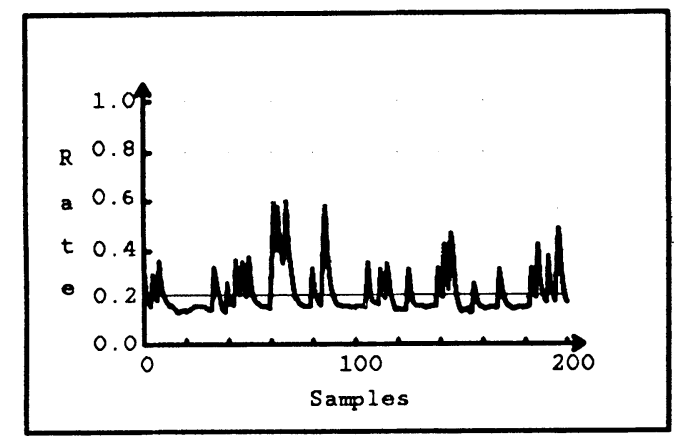

(c)

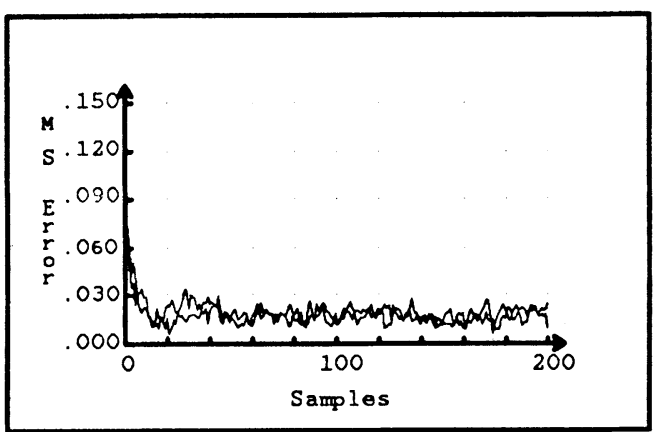

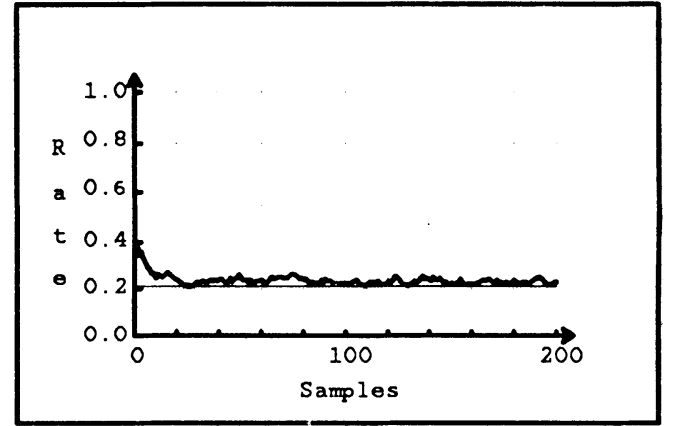

(b)

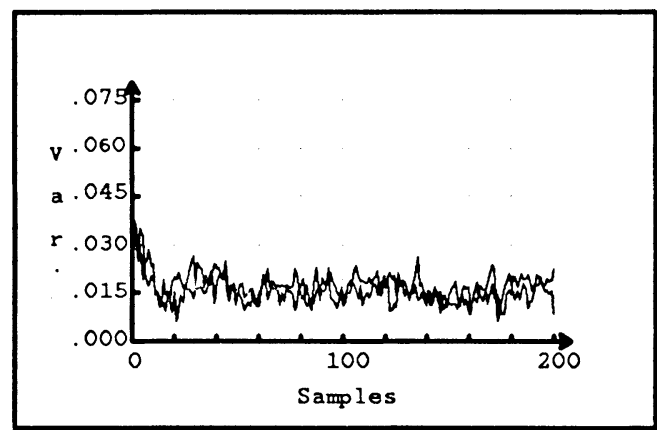

(d)

Fig. 4. Estimate of marginal probability $\pi_{3}(t)$. (a) Result of individual simulation. (b) Sample mean over Monte Carlo trials. (c) Mean-square estimation error. (d) Sample variance of estimate.

(a)

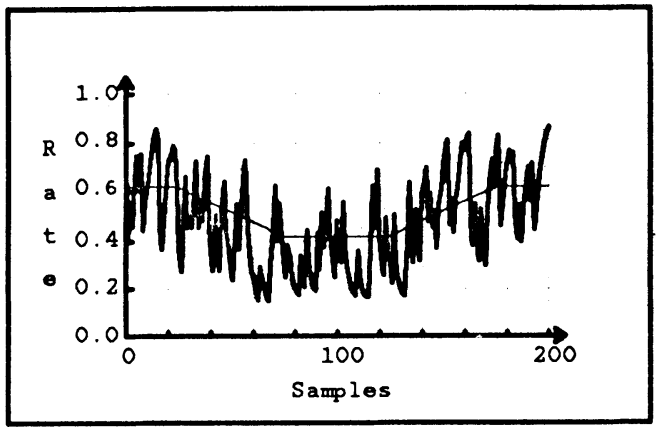

(c)

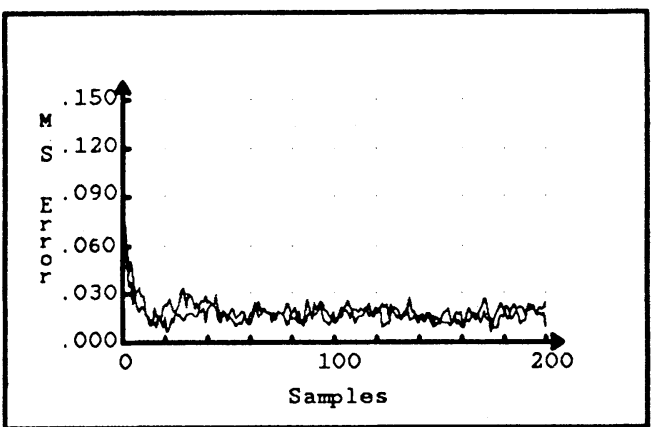

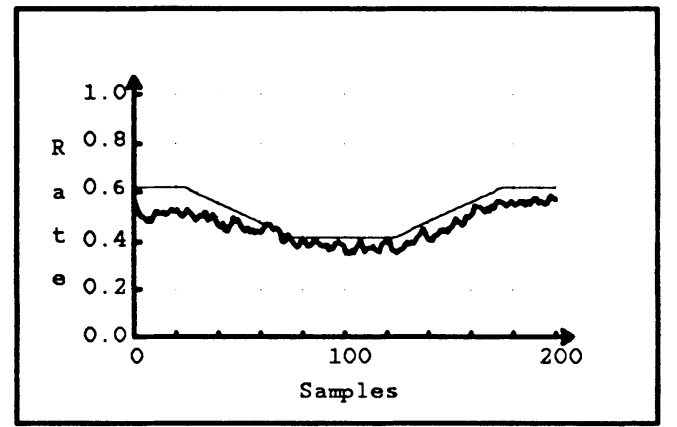

(b)

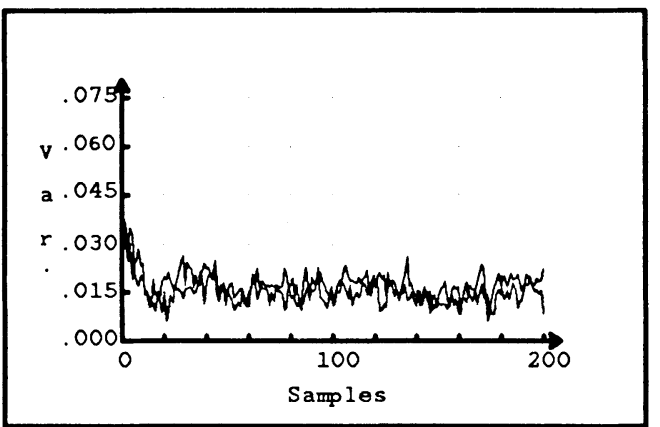

Fig. 5. Estimate of marginal probability $\pi_{1}(t)$. (a) Result of individual simulation. (b) Sample mean over Monte Carlo trials. (c) Mean-square estimation error. (d) Sample variance of estimate.

where $\lambda(t)$ is the true rate [either a marginal probability $\pi(t)$ or an element of $\boldsymbol{\Lambda}(t)]$ and $\hat{\lambda}(t, i)$ is its estimate for the $i$ th simulation.

We consider the performance of the algorithm in estimating both constant and time-varying marginal probabilities $\pi(t)$. Figs. 4-8 illustrate the effectiveness of the algorithm for a variety of test cases. The marginal prob- abilities for these cases are of the form

$$
\left[\begin{array}{l}
\pi_{1} \\
\pi_{2} \\
\pi_{3} \\
\pi_{4}
\end{array}\right]=\left[\begin{array}{l}
\pi_{1}(t) \\
0.2 \\
0.1 \\
0.1
\end{array}\right] .
$$


(a)

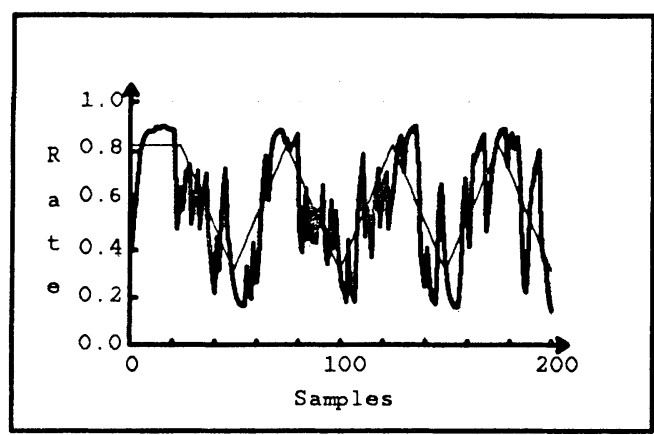

(c)

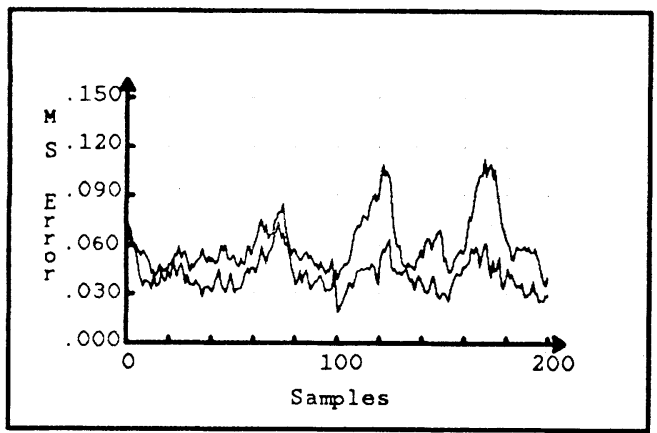

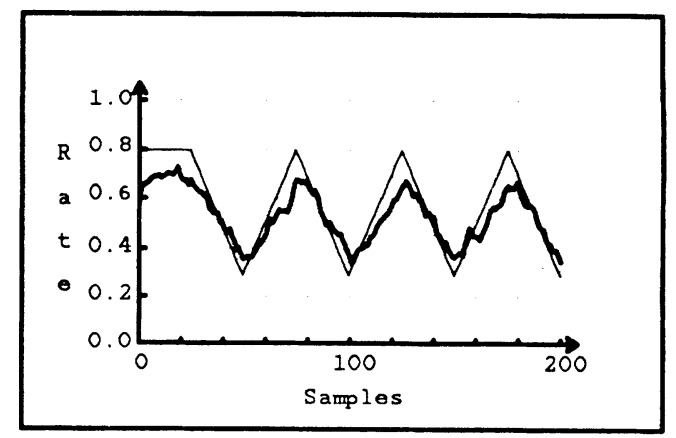

(b)

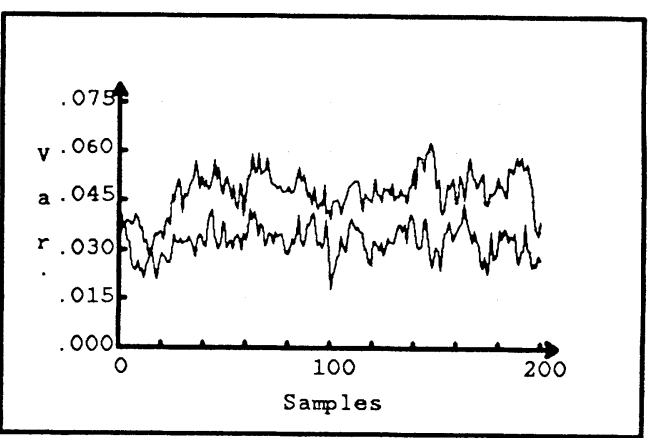

(d)

Fig. 6. Estimate of marginal probability $\pi_{1}(t)$. (a) Result of individual simulation. (b) Sample mean over Monte Carlo trials. (c) Mean-square estimation error. (d) Sample variance of estimate.

(a)

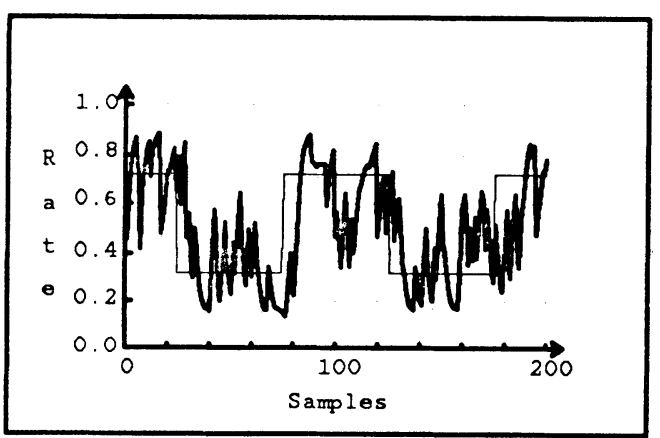

(c)

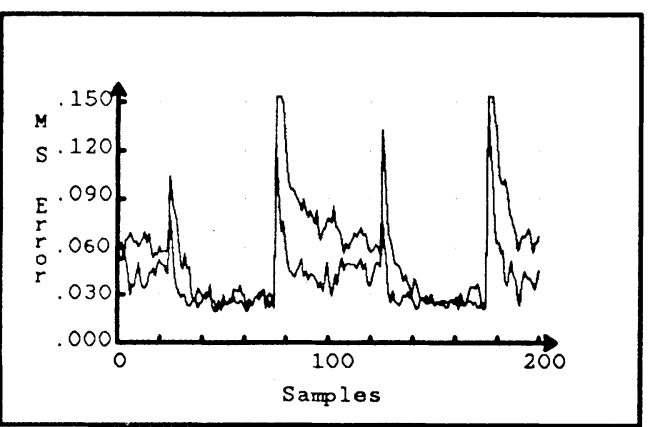

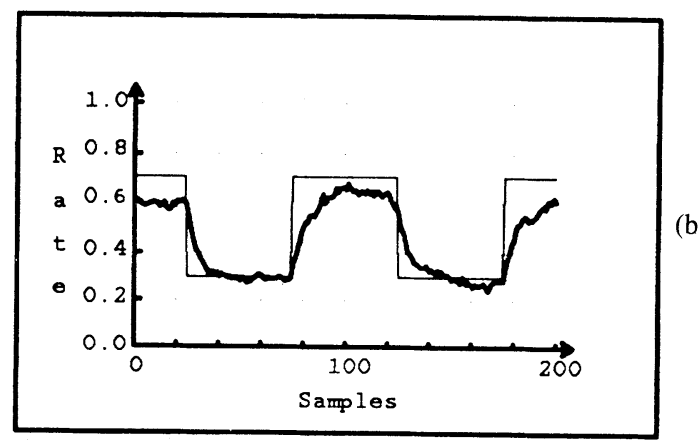

(b)

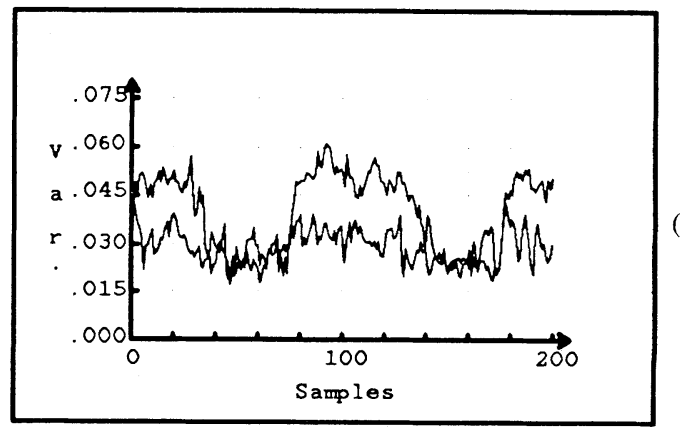

Fig. 7. Estimate of marginal probability $\pi_{1}(t)$. (a) Result of individual simulation. (b) Sample mean over Monte Carlo trials. (c) Mean-square estimation error. (d) Sample variance of estimate.

The function $\pi_{1}(t)$ assumes several values, as indicated in the figures. In each of these figures, the upper left-hand plot is a random sample of the rate estimate taken from one of the Monte Carlo simulations; the upper right-hand plot is the corresponding sample mean of all the Monte Carlo trials; the lower left-hand plot shows the meansquare estimation error through time for the particular test case considered; and the lower right-hand plot is the sample variance of the estimate through time. In the upper plots, the thin lines represent the true rate $\pi$, and the boldfaced lines represent the estimate $\hat{\lambda}$; the signal-to-noise ratio (SNR) is $9.5 \mathrm{~dB}$. The two curves shown in each of the lower plots represent the estimation error and sample variance for SNR's of 6 and $9.5 \mathrm{~dB}$. In all cases, the cor- 
(a)

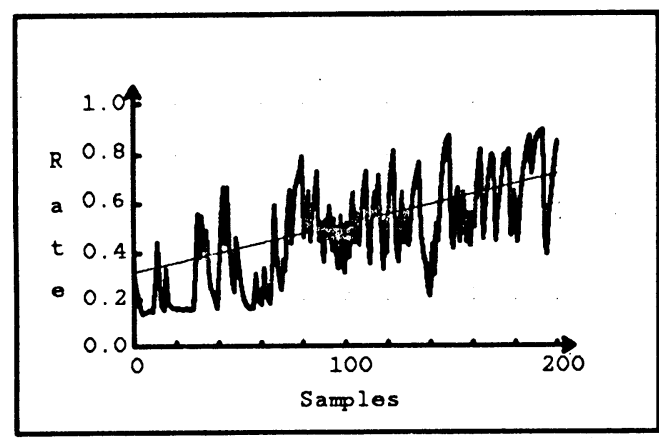

(c)

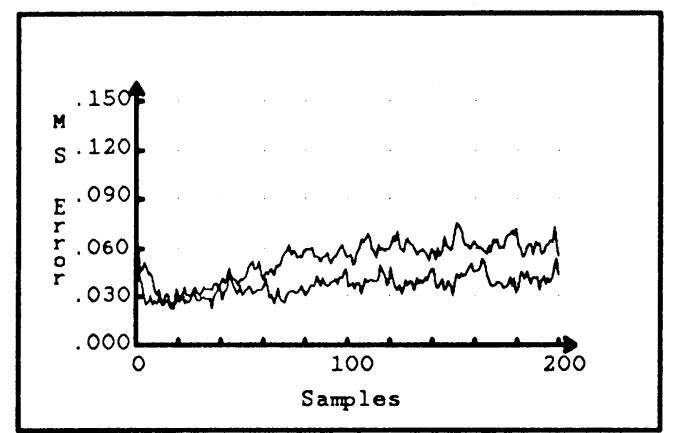

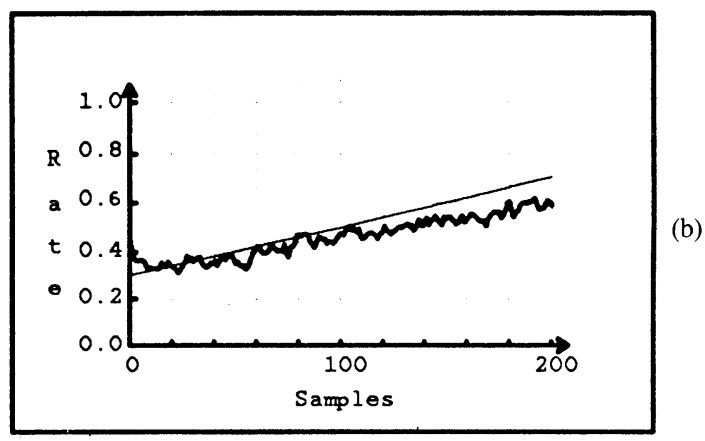

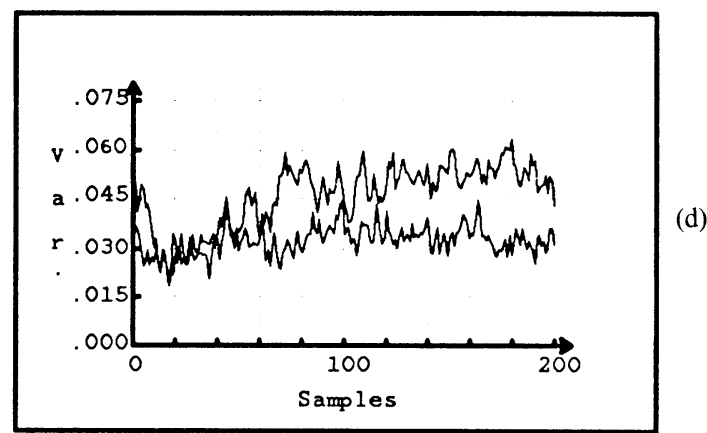

Fig. 8. Estimate of marginal probability $\pi_{1}(t)$. (a) Result of individual simulation. (b) Sample mean over Monte Carlo trials. (c) Mean-square estimation error. (d) Sample variance of estimate.

responding estimation error and sample variance are greater at the lower SNR.

It is evident from Figs. 4(a) and 4(b), which display results for $\pi_{3}$, that, as expected, the estimates are slightly biased. The mean-square estimation error and sample variance decrease to small steady-state values. Hence, the estimates converge despite the bias. An obvious characteristic of the constant bias estimation example illustrated in Fig. 4(a) is that although the average is fairly smooth, the individual trials exhibit considerable fluctuation. This phenomenon is due largely to the dynamical modeling assumption as represented by the transition matrix $\boldsymbol{Q}$. This model characterizes the rate as being time varying, which is not appropriate for the constant rate case. A more appropriate transition matrix would be to set $\boldsymbol{Q}$ to the identity matrix. This is not done in the simulation, however, since it is not a priori possible to know that the rate is constant. Thus, for this particular case, the problem is overmodeled. It should be mentioned in this context that no attempt was made to "tune", the dynamics model to effect an improved fit; the results that are displayed are typical. The reassuring aspect of this simulation is that, even though the estimator appears to get "pulled off"' the truth in the overmodeled case, it does not diverge.

The results of the time-varying cases, Figs. 5-8, are particularly promising. Even under severe conditions, such as in Figs. 6 and 7 where the rate varies rapidly with time, the estimates track with minimal delay and bias. The behavior of the mean-square estimation error in Fig. 7 is particularly interesting. The error, as expected, peaks at points where jumps in the rate occur and then decreases back to its steady-state value. This exhibits the stability of the algorithm in that sudden changes in parameter values only temporarily increase the estimation uncertainty. Note that the estimation error does not approach zero, as might be expected for the estimation of stationary priors. For the nonstationary case, the error will not, in general, be bounded away from zero. This behavior is essential since the filter gain is proportional to the covariance. An analogy is the Kalman filter, except that for this case the filter gain is a function of the estimated state. If the estimation error covariance is used as a measure of performance, this interpretation may result in pessimistic assessments. The reader is cautioned, however, against comparing these results to adaptive classification procedures associated with stationary parameters.

In Fig. 9, we show five selected examples of Monte Carlo sample means for estimates of individual elements of $\boldsymbol{\Lambda}(t)$, the prior probability distribution. The SNR in each of these simulations was $6 \mathrm{~dB}$. Again, the ability of the algorithm to track time-varying rates is clearly evident.

Figs. 10 and 11 demonstrate how the estimated bias is reduced and how the responsiveness of the estimator to rapidly changing rates is enhanced as signal power is increased. The three SNR's considered are 6, 9.5, and 12 $\mathrm{dB}$, and the plots shown are sample means of marginal probability estimates. These figures also demonstrate time-lag effects. As with any recursive filter (e.g., the Kalman filter), time lags are present when there are abrupt changes in the state that must be tracked. This phenomenon is due to the predictive nature of the estimator, and it can be seen from these figures that the size of the time lag decreases as the SNR increases. 
(a)
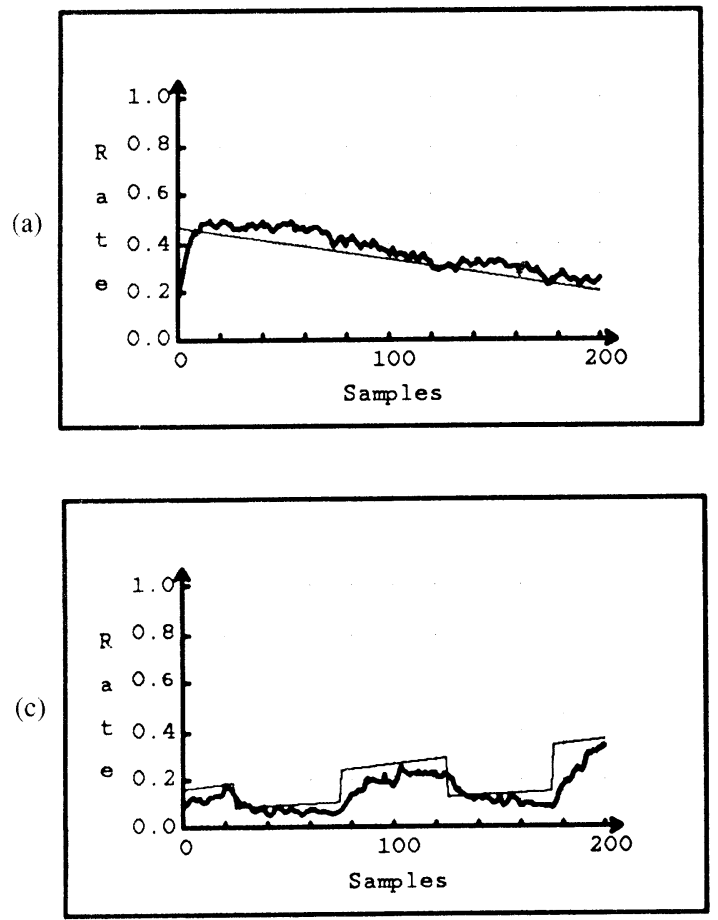

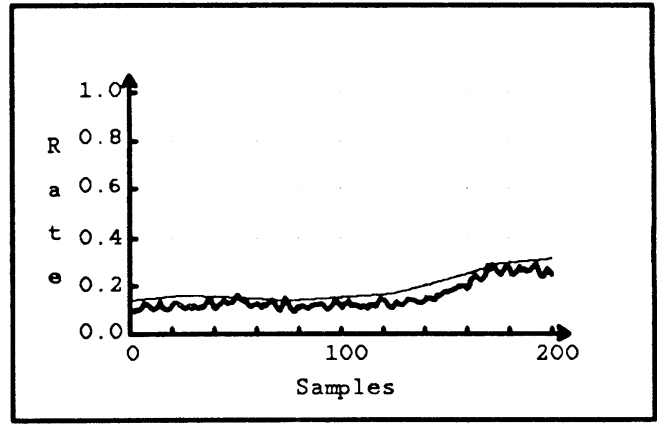

(b)

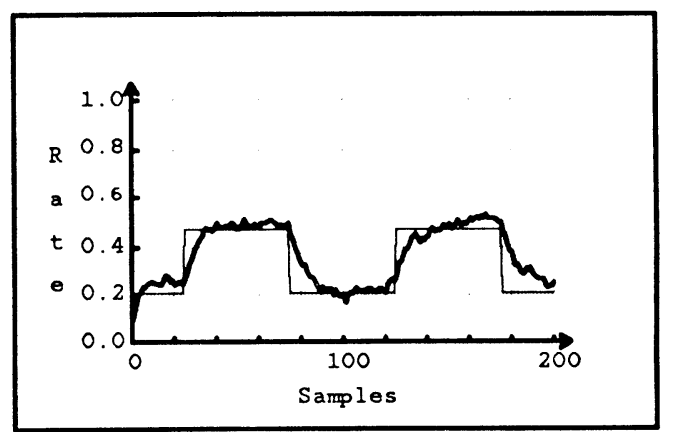

(d)

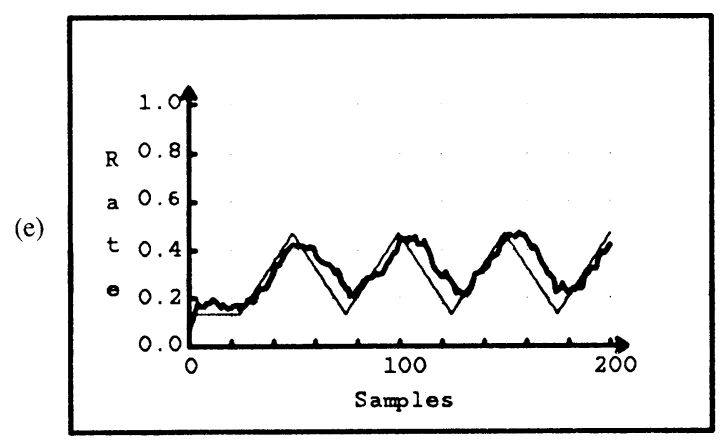

Fig. 9. Elements of $\boldsymbol{\Lambda}(t)$ at $6 \mathrm{~dB}$.

\section{Probability of Error}

As an indicator of the algorithm's performance, Figs. 12 and 13 show the probability of error versus SNR for the decision-directed empirical Bayes decision rule when testing against four hypotheses (a two-dimensional classifier). The curves on each plot correspond to the following three cases.

1) Prior probabilities and signal strengths are known exactly, i.e., the ideal classical Bayes case (plot indicated by thin line).

2) The signal strength is known exactly and priors are estimated by the recursive estimator (points on thicklined curve denoted by the symbol $\circ$ ).

3) Both priors and signal strength are estimated (points on thick-lined curve denoted by the symbol $\times$ ).

Note that in Fig. 13, only one curve is drawn for cases 2 and 3 since there is virtually no difference between them. It is seen that the Bayes risk of the decision-directed al- gorithm approaches the theoretical lower bound on probability of error as the SNR increases.

Our results demonstrate the ability of the decision-directed estimator to track accurately the prior probability distribution used in the empirical Bayes decision rule. The algorithm is especially effective in estimating time-varying rates, even under worst case scenarios (e.g., discontinuous jumps in the true prior, etc.). Since the presence of a bias in the estimates does not greatly affect algorithm performance, the large amount of computation that would be required to eliminate it is probably unjustified. Note also that the added complication of estimating $b_{i}(t)$ does not seriously degrade algorithm performance, at least for the eonstant-mean case considered.

\section{Conclusions}

The decision-directed empirical Bayes decision rule has been shown to perform effectively in a multivariate en- 


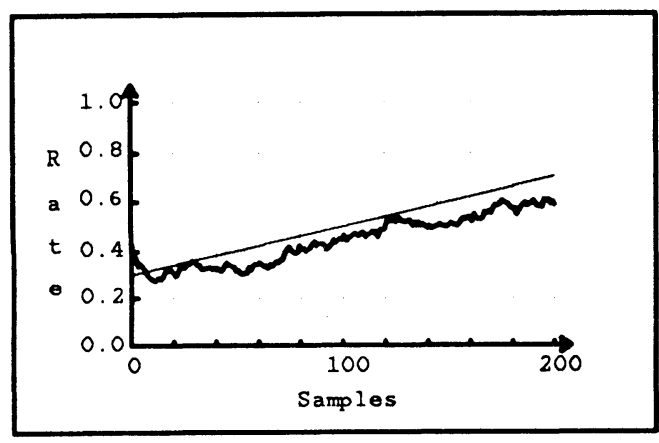

(a)

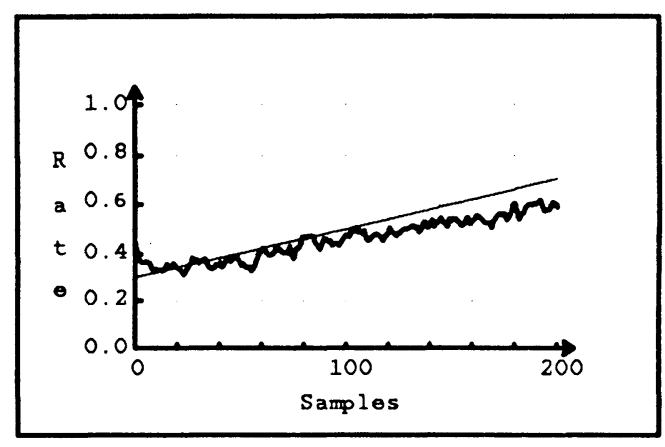

(b)

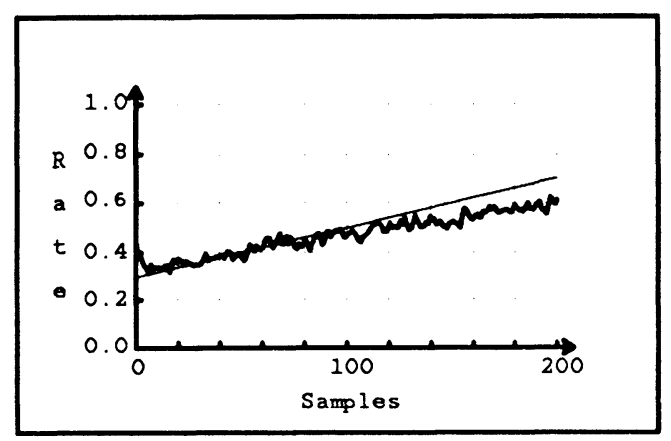

(c)

Fig. 10. Improvement of estimate with increasing SNR. (a) $6 \mathrm{~dB}$. (b) 9.5 dB. (c) $12 \mathrm{~dB}$.

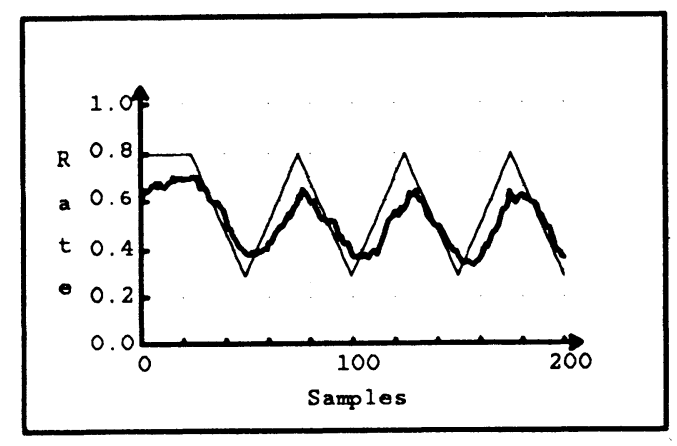

(a)

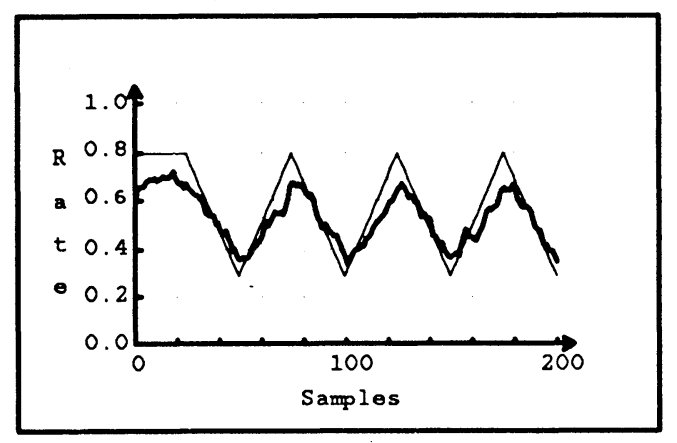

(b)

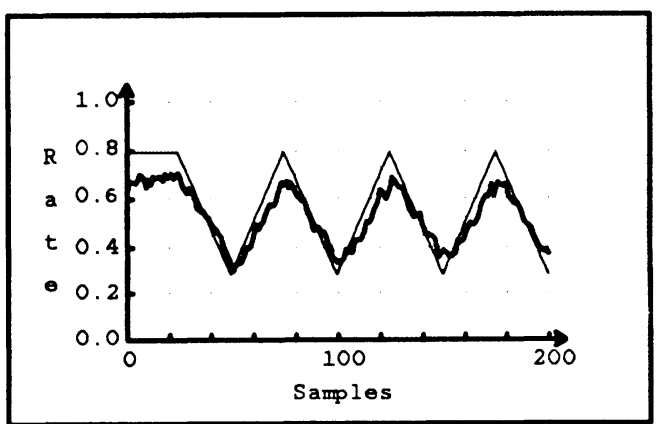

(c)

Fig. 11. Improvement of estimate with increasing SNR. (a) $6 \mathrm{~dB}$. (b) 9.5 dB. (c) $12 \mathrm{~dB}$. 


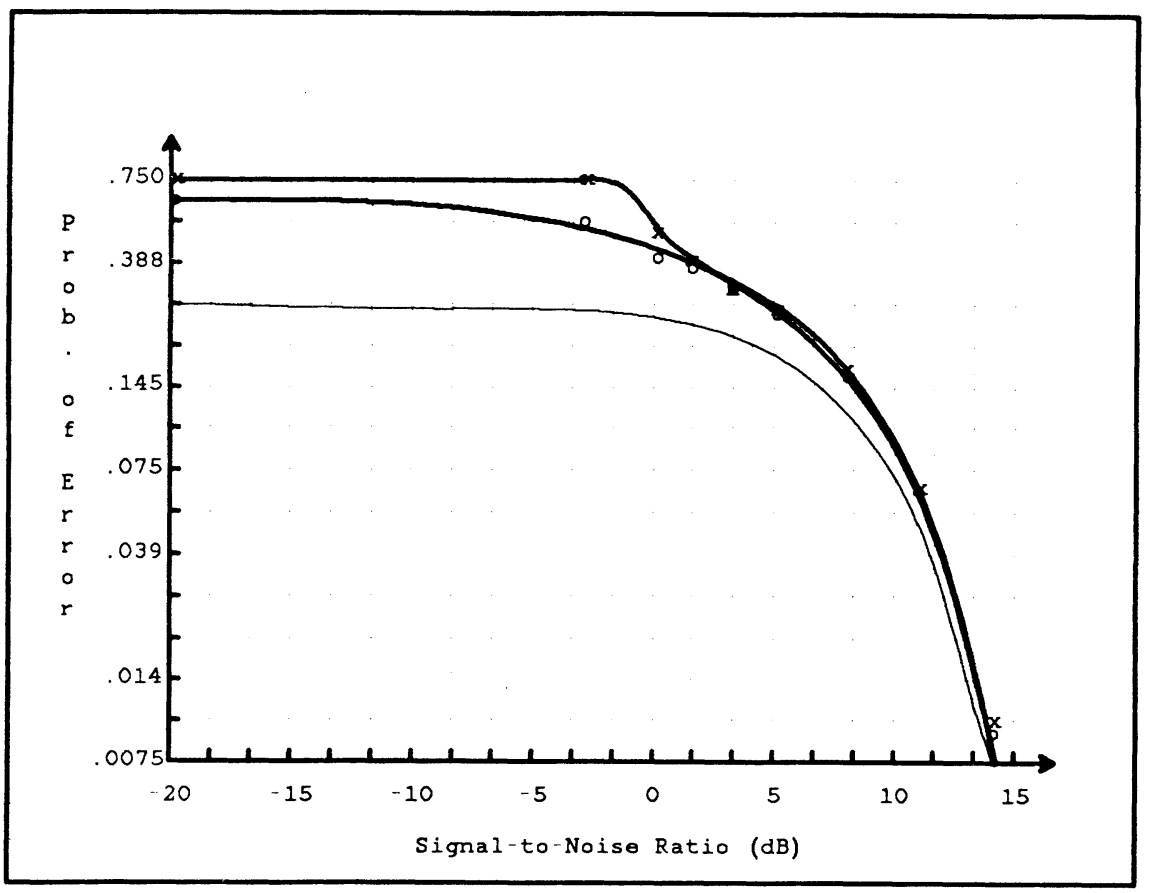

Fig. 12. Probability of error versus SNR for $\pi=\left[\begin{array}{lllll}0.72 & 0.18 & 0.08 & 0.02\end{array}\right]^{T}$.

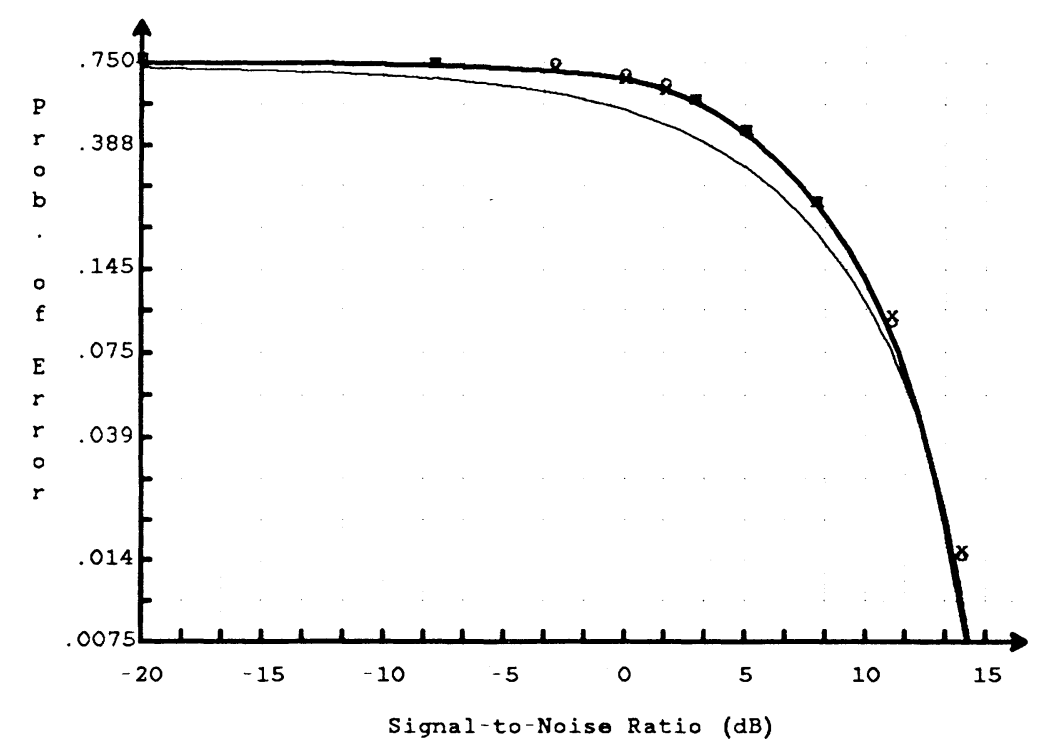

Fig. 13. Probability of error versus SNR for $\pi=\left[\begin{array}{llll}0.25 & 0.25 & 0.25 & 0.25\end{array}\right]^{T}$.

vironment. Using simulated data, a number of test scenarios have been conducted, and the performance of the decision rule has been evaluated. These controlled experiments have demonstrated that the detector effectively estimates the prior multivariate distribution of the signal environment. The problem of runaway is negligible in these simulations, but there is a bias on the estimates. This bias is expected and can be removed with further processing. There are a number of possible ways to eliminate or reduce the bias. The most direct way is to directly calculate the bias, but this involves a significant increase in the computational burden. An alternative would be to effect bias correction via a table lookup procedure and interpolate for the bias. This second procedure would not eliminate the bias completely, but would reduce it significantly. An additional alternative is to simply ignore the bias, which is indeed a viable approach. It is evident that as the true rates approach one or unity, the bias problem increases, whereas for rates near one-half, the bias problem is negligible. 
The decision-directed rule effectively tracks the timevarying prior distributions. A number of time-varying prior distributions have been simulated, and it has been shown that these rates may be effectively tracked with a decision-directed approach. The most obvious feature of these estimated rates is a time lag, which is a feature typical of real-time processing. The estimator cannot look ahead to the the future, and consequently, a few samples are required to lock on to the new rate. This lagging phenomenon cannot be eliminated with real-time processing; only non-real-time processing involving a smoothing algorithm is capable of removing such phenomena.

The performance of the decision-directed empirical Bayes detector is compared to the standard Bayes case where the prior is exactly known. It is shown that as the SNR increases, the performance as measured by the total probability of error for the empirical Bayes approach approaches that of the standard case. A striking aspect of the simulations studied is that the additional complication of estimating the signal parameters (the mean in the cases studied) does not significantly change the performance of the detector.

\section{NOMENCLATURE}

$\begin{array}{ll}a, b & \text { Scale and bias error } \\ B & \text { Matrix of signal means } \\ B_{t} & \sigma \text {-field generated by all factors that affect the } \\ & \text { distribution } N(t) \text { at time } t\end{array}$

$D, D \quad$ Decision function

$f(\cdot \mid \cdot)$ Class-conditional density function

$\mathcal{F}_{t} \quad \sigma$-field generated by decisions up to time $t$

$H \quad$ Hypothesis

$m \quad$ Dimension of signal space

$M \quad$ Dimension of decision problem

MD Martingale difference process

$N, \boldsymbol{N} \quad$ Discrete-time decision point process

$p_{N(t)} \quad$ Joint distribution of $N(t)$

$Q, q_{i j} \quad$ Markov chain state transition probabilities

$\boldsymbol{r} \quad$ Columns of $\boldsymbol{R}$

$\boldsymbol{R} \quad$ Matrix of Markov chain vectors

$s \quad$ Columns of $S$

$S \quad$ Classification set

$\boldsymbol{S} \quad$ Rate distribution matrix

$t \quad$ Time

$\boldsymbol{u} \quad$ State transition martingale difference process with respect to $B$

$v \quad$ Gaussian noise vector

$\boldsymbol{w}$ Decision martingale difference process with respect to $B$

$x, \boldsymbol{x}$ Indicator function for vector Markov chain state

$\boldsymbol{Y} \quad$ Signal space

$\boldsymbol{y} \quad$ Signal vector

$\alpha, \boldsymbol{\alpha} \quad$ Binary number, vector

$\beta \quad$ Element of decision process

$\delta \quad M$-ary decision function

$\lambda, \lambda$ Conditional probabilities (rates), rate vectors

$\Lambda \quad$ Vector of rates $\eta$

Decision martingale difference with respect to $\mathfrak{F}$

$\boldsymbol{\mu} \quad$ State transition martingale difference with respect to $\mathcal{F}$

$v \quad$ Signal vector

$\pi, \pi \quad$ A priori distribution

II $\quad$ Rate estimation error covariance

$\rho, \boldsymbol{\rho} \quad$ Elements of Markov chain vector

\section{REFERENCES}

[1] H. Robbins, "Asymptotically subminimax solutions of compound statistical decision problems," in Proc. Second Berkley Symp. Math. Stat. Probability, Berkeley, CA, University of California Press, 1950, pp. 131-148.

[2] - "An empirical Bayes approach to statistics," in Proc. Third Berkley Symp. Math. Stat. Probability, Berkeley, CA, University of California Press, 1955, pp. 157-164.

[3] E. Samuel, "Asymptotic solutions of sequential compound decision problem," Ann. Math. Stat., vol. 34, pp. 1079-1094, 1963.

[4] J. Neyman, "Two breakthroughs in the theory of statistical decision making," Rev. Inst. Int. Stat., vol. 30, pp. 11-27, 1962.

[5] D. Middleton and R. Esposito, "Simultaneous optimum detection and estimation of signals in noise," IEEE Trans. Inform. Theory, vol. IT14, pp. 434-444, May 1968.

[6] A. Fredriksen, D. Middleton, and D. VandeLinde, "Simultaneous signal detection and estimation under multiple hypotheses," IEEE Trans. Inform. Theory, vol. IT-18, pp. 607-614, Sept. 1972.

[7] H. J. Scudder, III, "Probability of error of some adaptive patternrecognition machines," IEEE Trans. Inform. Theory, vol. IT-11, pp. 363-371, July 1965.

[8] E. A. Patrick and J. P. Costello, “Asymptotic probability of error using two decision-directed estimators for two unknown mean vectors," IEEE Trans. Inform. Theory, vol. IT-14, pp. 160-162, Jan. 1968.

[9] E. A. Patrick, "On a class of unsupervised estimation problems,", IEEE Trans. Inform. Theory, vol. IT-14, pp. 407-415, May 1968.

[10] E. A. Patrick, J. P. Costello, and F. C. Monds, "Decision-directed estimation of a two-class decision boundary," IEEE Trans. Comput., vol. C-19, pp. 197-205, Mar. 1970.

[11] J. G. Proakis, P. R. Drouilhet, Jr., and R. Price, "Performance of coherent detection systems using decision-directed channel measurement,"' IEEE Trans. Commun. Syst., vol. CS-12, pp. 54-63, Mar. 1964.

[12] R. W. Lucky, "'Techniques for adaptive equalization of digital communications," Bell Syst. Tech. J., vol. 45, pp. 255-286, Feb. 1966.

[13] S. C. Schwartz and A. Katopis, "Modified stochastic approximation to enhance unsupervised learning," in Proc. 1977 Conf. Decision Contr., San Francisco, CA, 1977, pp. 1067-1079.

[14] Ya. Z. Tsypkin and G. K. Kel'mans, "The adaptive Bayes procedure," Problemy Peredachi Informatsii, vol. 6, no. 1, pp. 52-59, 1970.

[15] N. Ula, "An asymptotic optimal empirical Bayes estimation scheme," IEEE Trans. Automat. Contr., vol. AC-22, pp. 991-992, Dec. 1977.

[16] A. K. C. Wong and T. S. Liu, "A decision-directed clustering algorithm for discrete data,"' IEEE Trans. Comput., vol. C-26, pp. 7582, Jan. 1977

[17] T. Y. Young and A. A. Farjo, "On decision-directed estimation and stochastic approximation," IEEE Trans. Inform. Theory, vol. IT-18, pp. 671-673, Sept. 1972.

[18] R. J. McAulay and E. Denlinger, "A decision-directed adaptive tracker," IEEE Trans. Aerospace Electron. Syst., vol. AES-9, pp. 229-236, Mar. 1973.

[19] U. Peters, "Detection with prediction and decision feedback," in Signal Processing II: Theories and Applications, H. W. Schüssler, Ed. Amsterdam, The Netherlands: Elsevier Science, 1983, pp. 544546.

[20] A. Sano and S. Adachi, "New decision-directed binary detector with looking-ahead structure and fast adaptive algorithms, " in Signal Processing II: Theories and Applications, H. W. Schüssler, Ed. Amsterdam, The Netherlands: Elsevier Science, pp. 547-550

[21] W. D. Gregg and J. C. Hancock, "An optimum decision-directed scheme for Gaussian mixtures," IEEE Trans. Inform. Theory, vol. IT-14, pp. 451-461, May 1968. 
[22] L. D. Davisson and S. C. Schwartz, "Analysis of a decision-directed receiver with unknown priors," IEEE Trans. Inform. Theory, vol. IT16, pp. 270-276, May 1970.

[23] A. Katopis and S. C. Schwartz, "Decision-directed learning using stochastic approximation," in Proc. 3rd Annu. Pittsburgh Conf. Modeling Simulation, Univ. Pittsburgh, Pittsburgh, PA, Apr. 1972, pp. 473-481.

[24] D. Kazakos and L. D. Davisson, "An improved decision-directed detector," IEEE Trans. Inform. Theory, vol. IT-26, pp. 113-116, Jan. 1980.

[25] S. C. Schwartz and L. D. Davisson, "Analysis of a decision-directed receiver for a Markov sequence with unknown transition probabilities," Problemy Peredachi Informatsii, vol. 6, no. 2, pp. 92-86, Apr.-June 1970 .

[26] W. C. Stirling, "'Simultaneous Jump Excitation Modeling and System Parameter Estimation," Ph.D. dissertation, Stanford Univ., Stanford, CA, 1983.

[27] W. C. Stirling and M. Morf, “'A new decision-directed algorithm for nonstationary priors," IEEE Trans. Automat. Contr., vol. AC-29, pp. 928-930, Oct. 1984

[28] W. C. Stirling, "Simultaneous system identification and decision-directed detection and estimation of jump inputs to linear systems,", IEEE Trans. Automat. Contr., vol. AC-32, Feb. 1987.

[29] J. L. Doob, Stochastic Processes. New York: Wiley, 1953.

[30] R. S. Liptser and A. N. Shiryayev, Statistics of Random Processes I. New York: Springer-Verlag, 1977.

[31] A. Segall, "Stochastic processes in estimation theory,"' IEEE Trans. Inform. Theory, vol. IT-22, pp. 275-286, May 1976.

[32] —, "Recursive estimation from discrete-time point processes," IEEE Trans. Inform. Theory, vol. IT-22, pp. 422-431, July 1976.

[33] A. L. Swindlehurst, "Analysis of a Multivariate Decision-Directed Empirical Bayes Classifier,' Master's thesis, Brigham Young Univ., Provo, UT, 1986

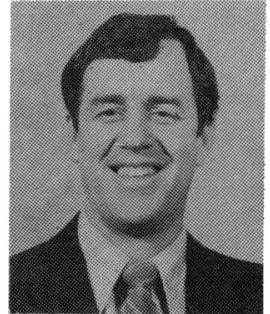

Wynn C. Stirling was born in St. George, UT, in 1945 . He received the B.A. degree in mathematics and the M.S. degree in electrical engineering from the University of Utah, Salt Lake City, UT, in 1969 and 1971, respectively, and the Ph.D. degree in electrical engineering from Stanford University, Stanford, CA, in 1983.

From 1972 to 1975 he was employed by Rockwell International Corporation, Anaheim, CA, and from 1975 to 1984 he was with ESL, Inc., Sunnyvale, CA. Since 1984 he has been an Associate Professor in the Department of Electrical and Computer Engineering at Brigham Young University, Provo, UT. His research interests include detection and estimation theory, information theory, and stochastic processes.

Dr. Stirling is a member of Phi Beta Kappa and Tau Beta Pi.

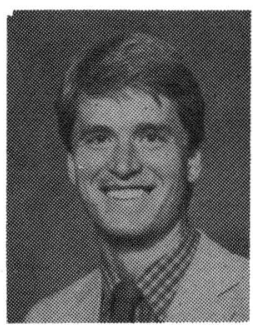

A. Lee Swindlehurst was born on March 10, 1960 , in Boulder City, NV. He received the B.S. and M.S. degrees in electrical engineering from Brigham Young University, Provo, UT, in 1985 and 1986, respectively.

He was awarded an Office of Naval Research Graduate Fellowship for 1985-1988 and is presently a candidate for the Ph.D. degree in electrical engineering at Stanford University, Stanford, CA, where he is associated with the Information Systems Laboratory. His research interests include detection and estimation theory, array signal processing, and adaptive algorithms. 